https://helda.helsinki.fi

\title{
Renormalization of Generalized KPZ Equation
}

\section{Kupiainen, Antti}

2017-02

Kupiainen , A \& Marcozzi , M 2017 , ' Renormalization of Generalized KPZ Equation ' , Journal of Statistical Physics, vol. 166 , no. 3-4 , pp. 876-902 . https://doi.org/10.1007/s10955-016-1636-3

http://hdl.handle.net/10138/313117

https://doi.org/10.1007/s10955-016-1636-3

acceptedVersion

Downloaded from Helda, University of Helsinki institutional repository.

This is an electronic reprint of the original article.

This reprint may differ from the original in pagination and typographic detail.

Please cite the original version. 


\title{
RENORMALIZATION OF GENERALIZED KPZ EQUATION
}

\author{
ANTTI KUPIAINEN AND MATTEO MARCOZZI
}

\begin{abstract}
We use Renormalization Group to prove local well posedness for a generalized KPZ equation introduced by H. Spohn in the context of stochastic hydrodynamics. The equation requires the addition of counter terms diverging with a cutoff $\epsilon$ as $\epsilon^{-1}$ and $\log \epsilon^{-1}$
\end{abstract}

\section{INTRODUCTION}

Nonlinear stochastic PDE's driven by a space time white noise have been under intensive study in recent years [1, 2, 3, 4, 5]. These equations are of the form

$$
\partial_{t} u=\Delta u+V(u)+\Xi
$$

where $u(t, x) \in \mathbb{R}^{n}$ is defined on $\Lambda \subset \mathbb{R}^{d}, V(u)$ is a function of $u$ and possibly its derivatives which can also be non-local and $\Xi$ is white noise on $\mathbb{R} \times \Lambda$, formally

$$
\mathbb{E} \Xi_{\alpha}\left(t^{\prime}, x^{\prime}\right) \Xi_{\beta}(t, x)=\delta_{\alpha \beta} \delta\left(t^{\prime}-t\right) \delta\left(x^{\prime}-x\right) .
$$

In order to be defined these equations in general require renormalization. One first regularizes the equation by e.g. replacing the noise by a mollified version $\Xi^{(\epsilon)}$ which is smooth on scales less than $\epsilon$ and then replaces $V$ by $V^{(\epsilon)}=V+W^{(\epsilon)}$ where $W^{(\epsilon)}$ is an $\epsilon$-dependent "counter term". One attempts to choose this so that solutions converge as $\epsilon \rightarrow 0$.

The rationale of such counterterms is that although they diverge as $\epsilon \rightarrow 0$ their effect on solutions on scales much bigger than $\epsilon$ is small. They are needed to make the equation well posed in small scales but they disturb it little in large scales.

Such a phenomenon is familiar in quantum field theory. For instance in quantum electrodynamics the "bare" mass and charge of the electron have to be made cutoff dependent so as to have cutoff independent measurements at fixed scales. The modern way to do this is to use the Renormalization Group (RG) method which constructs a one parameter family of effective theories describing how the parameters of the theory vary with scale.

Such a RG method was applied to SPDE's in [5] for the case $n=1, d=3$ and $V(u)=u^{3}$. In that case $W^{(\epsilon)}=\left(a \epsilon^{-1}+b \log \epsilon\right) u$ and path wise solutions were constructed recovering earlier results by [1, 2]. In the present paper we consider the equations of Stochastic Hydrodynamics recently introduced by Spohn [6]. They give rise to the problem (11) with $n=3, d=1$ and

$$
V(u)=\left(\partial_{x} u, M \partial_{x} u\right)
$$

where $(\cdot, \cdot)$ denotes the standard inner product in $\mathbb{R}^{3}$ and $M=\left(M^{(1)}, M^{(2)}, M^{(3)}\right)$ with $M^{(i)}$ are symmetric matrices, so that (3) can be read component-wise as $V_{i}(u)=$

Date: October 17, 2018.

Supported by Academy of Finland. 
$\left(\partial_{x} u, M^{(i)} \partial_{x} u\right)$ for $i=1,2,3$. We construct path wise solutions in this case by taking

$$
W^{(\epsilon)}=a \epsilon^{-1}+b \log \epsilon .
$$

The case $n=1$ is the KPZ equation and this was constructed before by Hairer [7]. In that case $b=0$. For a generic $M_{\alpha \beta \gamma}$ in (3) $b \neq 0$. This counter term is third order in the nonlinearity as will be explained below. Thus in this case the simple Wick ordering of the nonlinearity does not suffice to make the equation well posed.

The content of the paper is as follows. In section 2 we define the model and state the result. The RG formalism is set up in a heuristic fashion in Section 3. Section 4 discusses the leading perturbative solution and sets up the fixed point problem for the remainder. Section 5 states the estimates for the perturbative noise contributions and in Section 6 the functional spaces for RG are defined and the fixed point problem solved. The main result is proved in Section 7. Finally in Sections 8 estimates for the covariances of the various noise contributions are proved.

\section{The Regularized EQUATION AND MAIN RESUlt}

We consider the equation (1) with $u(t, x)$ defined on $(t, x) \in \mathbb{R} \times \mathbb{T}$ and nonlinearity given by (3). We study its integral form

$$
u=G *\left[(V(u)+\Xi) \mathbf{1}_{t \geq 0}\right]+e^{t \Delta} u_{0}
$$

where $G(t, x)=e^{t \Delta}(x, 0)$ and $u_{0}$ is the initial condition. In this paper we consider a random initial condition of Brownian type. Concretely we take $u_{0}$ the stationary solution to the linear problem $V=0$ which is the Gaussian random field with covariance

$$
\mathbb{E} u_{0}(x) u_{0}(y)=\sum_{n \in \mathbb{Z} \backslash\{0\}} \frac{e^{2 \pi i n(x-y)}}{2(2 \pi n)^{2}} .
$$

$\Xi$ is taken to be the white noise with vanishing spatial average i.e.

$$
\Xi(t, x)=\sum_{n \in \mathbb{Z} \backslash\{0\}} e^{2 \pi i n x} \dot{b}_{n}(t)
$$

with $b_{n}=\bar{b}_{-n}$ independent complex Brownian motions. Thus (4) can be written in the form

$$
u=G *\left(V(u) \mathbf{1}_{t \geq 0}+\Xi\right)
$$

Instead of mollifying the noise we regularize the convolution by considering

$$
u=G_{\epsilon} *\left(V^{(\epsilon)}(u)+\Xi\right)
$$

where

$$
G_{\epsilon}(t, x)=e^{t \Delta}(x, 0)\left(1-\chi\left(\epsilon^{-2} t\right)\right)
$$

with $\chi \geq 0$ being a smooth bump, $\chi(t)=1$ for $t \in[0,1]$ and $\chi(t)=0$ for $t \in[2, \infty)$ and

$$
V^{(\epsilon)}(u)=\left[(\partial u, M \partial u)+C_{\epsilon}\right] \mathbf{1}_{t \geq 0}
$$

We look for $C_{\epsilon}$ such that (6) has a unique solution $u^{(\epsilon)}$ which converges as $\epsilon \rightarrow 0$ to a non trivial limit. Note that $G_{\epsilon} * \Xi$ is a.s. smooth.

Our main result is 
Theorem 1. There exits $C_{\epsilon}$ s.t. the following holds. For almost all realizations of the white noise $\Xi$ there exists $t(\Xi)>0$ such that the equation (6) has for all $\epsilon>0$ a unique smooth solution $u^{(\epsilon)}(t, x), t \in[0, t(\Xi)]$ and there exists $u \in \mathcal{D}^{\prime}([0, t(\Xi)] \times \mathbb{T})$ such that $u^{(\epsilon)} \rightarrow u$ in $\mathcal{D}^{\prime}([0, t(\Xi)] \times \mathbb{T})$. The limit $u$ is independent of the regularization function $\chi$.

Remark 2. We will find that the renormalization parameter is given by

$$
C_{\epsilon}=m_{1} \epsilon^{-1}+m_{2} \log \epsilon^{-1}+m_{3}
$$

where the constants $m_{1}$ and $m_{3}$ depend on $\chi$ whereas the $m_{2}$ is universal i.e. independent on $\chi$. Furthermore, $m_{2}=0$ if $M_{\beta \gamma}^{(\alpha)}$ is totally symmetric in the three indices.

\section{Renormalization group}

The regularized equation (6) can be viewed as dealing with spatial scales larger than $\epsilon$. The idea of the Renormalization Group (RG) is to try to increase this small scale cutoff by deriving effective equations with larger cutoffs. This will be done inductively by going from scale $\ell$ to scale $L \ell$ with with $L$ fixed. One such step is called the RG transformation. It is useful to utilize the underlying scale invariance of the linear part of the equation and rescale at each step the small scale cutoff to unity. To do this define the space time scaling $s_{\mu}$ by

$$
\left(s_{\mu} f\right)(t, x)=\mu^{-\frac{1}{2}} f\left(\mu^{2} t, \mu x\right)
$$

and set

$$
\varphi=s_{\epsilon} u \text {. }
$$

Note that $\varphi$ is defined on $\mathbb{R} \times \epsilon^{-1} \mathbb{T}$. By a simple change of variables in (6) we obtain

$$
\varphi=G_{1} *\left(v^{(\epsilon)}(\varphi)+\xi\right)
$$

where

$$
v^{(\epsilon)}(\varphi):=\epsilon^{\frac{1}{2}}\left(\partial_{x} \varphi, M \partial_{x} \varphi\right)+\epsilon^{\frac{3}{2}} C_{\epsilon}
$$

and $\xi:=\epsilon^{2} s_{\epsilon} \Xi$ is equal in law with the white noise on $\mathbb{R} \times \epsilon^{-1} \mathbb{T}$ (we keep the convention that $v^{(\epsilon)}(\varphi)=0$ for $\left.t<0\right)$.

We note that in these dimensionless variables the small scale cutoff is unity and the strength of the nonlinearity is small, $\epsilon^{\frac{1}{2}}$ i.e. the model is subcritical. However, the price we pay is that we need to consider times of order $\epsilon^{-2}$ and spatial box of size $\epsilon^{-1}$.

Let us now attempt to increase the cutoff $\epsilon$. Fix $L>1$ and decompose

$$
G_{1}=G_{L^{2}}+\left(G_{1}-G_{L^{2}}\right)
$$

and

$$
\varphi=\varphi_{1}+\varphi_{2} .
$$

Then (11) is equivalent to the pair of equations

$$
\begin{aligned}
\varphi_{1} & =G_{L^{2}}\left(v^{(\epsilon)}\left(\varphi_{1}+\varphi_{2}\right)+\xi\right) \\
\varphi_{2} & =\left(G_{1}-G_{L^{2}}\right)\left(v^{(\epsilon)}\left(\varphi_{1}+\varphi_{2}\right)+\xi\right) .
\end{aligned}
$$

$\varphi_{1}$ can be thought of living on scales $\geq L$ and $\varphi_{2}$ on scales $\in[1, L]$. Rescale now back to unit cutoff. Let $s:=s_{L^{-1}}$ and set

$$
\varphi_{1}=s \varphi^{\prime}, \quad \varphi_{2}=s \zeta .
$$

Then

$$
\varphi=s\left(\varphi^{\prime}+\zeta\right)
$$


with $\varphi^{\prime}, \zeta$ solutions to

$$
\begin{aligned}
\varphi^{\prime} & =G_{1} *\left(S v^{(\epsilon)}\left(\varphi^{\prime}+\zeta\right)+\xi\right) \\
\zeta & =\Gamma *\left(S v^{(\epsilon)}\left(\varphi^{\prime}+\zeta\right)+\xi\right)
\end{aligned}
$$

where we defined the scaling operation

$$
(S v)(\varphi)=L^{2} s^{-1} v(s \varphi)
$$

and denoted

$$
\Gamma(t, x):=e^{t \Delta}(x, 0)\left(\chi(t)-\chi\left(L^{2} t\right)\right) .
$$

Note that $\Gamma$ involves scales between $L^{-1}$ and 1 so that the equation (15) turns out to be tractable: its solution $\zeta$ is a function $\zeta\left(\varphi^{\prime}\right)$ of $\varphi^{\prime}$. Plugging this into the large scale equation (14) yields

$$
\varphi^{\prime}=G_{1} *\left(\mathcal{R} v^{(\epsilon)}\left(\varphi^{\prime}\right)+\xi\right)
$$

where the new nonlinearity $\mathcal{R} v^{(\epsilon)}$ is defined by

$$
\mathcal{R} v^{(\epsilon)}\left(\varphi^{\prime}\right)=S v^{(\epsilon)}\left(\varphi^{\prime}+\zeta\left(\varphi^{\prime}\right)\right) .
$$

$\mathcal{R}$ is the Renormalization Group map: given a function $v$ mapping a field $\varphi(t, x)$ to a field $v(\varphi)(t, x)$ we obtain a new function $\mathcal{R} v$ by solving the small scale equation. Using (15) in (18) we may write the latter as an equation to determine $\mathcal{R} v$ :

$$
\mathcal{R} v(\varphi)=S v(\varphi+\Gamma *(\mathcal{R} v(\varphi)+\xi)) .
$$

We will set up the functional spaces where (19) is solved in Section 6. At this point let us see on a formal level how the solution of the original SPDE is reduced to the study of the map $\mathcal{R}$. To do this it is convenient to take the cutoff $\epsilon$ as

$$
\epsilon=L^{-N}
$$

so that we are interested in the limit $N \rightarrow \infty$. With a slight abuse of notation, denote $v^{(\epsilon)}$ by $v^{(N)}$ and define inductively

$$
v_{n-1}^{(N)}:=\mathcal{R} v_{n}^{(N)} .
$$

for $n=N, N-1, \ldots$

We call $v_{n}^{(N)}$ the effective potential at scale $L^{-n}$ starting with cutoff $L^{-N}$. They are related to each other by the iteration

$$
v_{n-1}^{(N)}(\varphi)=S v_{n}^{(N)}\left(\varphi+\Gamma_{n} *\left(v_{n-1}^{(N)}(\varphi)+\xi_{n-1}\right)\right)
$$

where we denote explicitly the dependence of the noise on the scale:

$$
\xi_{n}:=L^{-2 n} s^{-n} \Xi .
$$

$\xi_{n}$ equals in law the white noise in $\mathbb{R} \times L^{n} \mathbb{T}$. $\Gamma_{n}$ is the operator (16) on $\mathbb{R} \times L^{n} \mathbb{T}$.

Remark 3. The definition of $\mathcal{R}$ involves the scale $n$ i.e. the size $L^{n}$ of the spatial box where the heat kernel in (16) is defined. We suppress this dependence in the notation unless we want to emphasize it.

From (13) we infer that solutions to the equations $v$ and $v^{\prime}=\mathcal{R} v$ are related by

$$
\varphi=s\left(\varphi^{\prime}+\Gamma *\left(v^{\prime}\left(\varphi^{\prime}\right)+\xi\right)\right) .
$$

This leads to an iterative construction of the solution as follows. Suppose $\varphi_{n}$ solves the effective equation

$$
\varphi_{n}=G_{1} *\left(v_{n}^{(N)}\left(\varphi_{n}\right)+\xi_{n}\right) .
$$


Then, the solution of the original equation (11) is given by

$$
\varphi=s^{-(N-n)} f_{n}^{(N)}\left(\varphi_{n}\right) .
$$

where the maps $f_{n}^{(N)}$ satisfy the induction

$$
f_{n-1}^{(N)}(\varphi)=L^{-2} S f_{n}^{(N)}\left(\varphi+\Gamma_{n} *\left(v_{n-1}^{(N)}(\varphi)+\xi_{n-1}\right)\right)
$$

with the initial condition

$$
f_{N}^{(N)}(\varphi)=\varphi
$$

Recalling (10) we conclude that the solution of the SPDE with cutoff $\epsilon$ is given by

$$
u=s^{n} f_{n}^{(N)}\left(\varphi_{n}\right) \text {. }
$$

Suppose now that (a) we can control the $v_{n}^{(N)}$ and $f_{n}^{(N)}$ for $n \geq m$, (b) we can solve (23) for $n=m$ on the time interval $[0,1]$ (c) the solution $\varphi_{m}$ is in the domain of $f_{m}^{(N)}$. Then (27) yields the solution of the SPDE on the time interval $\left[0, L^{-2 m}\right]$.

What determines the smallest $m$ so that (a)-(c) hold? This is determined by the realization of the noise $\Xi$. Indeed, the $v_{n}^{(N)}$ are random objects i.e. functions of the white noise $\Xi$. Let $\mathcal{E}_{m}$ be the event such that the above holds for all $N, n$ with $m \leq n \leq N$. We will show that almost surely $\mathcal{E}_{m}$ holds for some $m<\infty$. For a precise statement see Section 5 .

Equations (11), (22) and (25) involve the convolution operators $\Gamma_{n}$ and $G_{1}$ respectively. These operators are infinitely smoothing and their kernels have fast decay in space time. In particular the noise $\zeta=\Gamma_{n} * \xi_{n-1}$ entering equations (22) and (25) has a smooth covariance which has finite range in time and it has Gaussian decay in space. Hence the fixed point problem (22) turns out to be quite easy.

\section{Perturbative contributions}

The RG iteration we have defined is quite general: formally it holds for "arbitrary" nonlinearity $v$ (and in any dimension as well, with appropriate scaling $s$ ). In the case at hand $v$ is a function of $\partial_{x} \varphi$ so it pays to change variables and denote

$$
\phi:=\partial_{x} \varphi
$$

Denote also

$$
v_{n}^{(N)}(\varphi)=w_{n}^{(N)}(\phi)
$$

and redefine the scaling operation as

$$
(\mathfrak{s} \phi)(t, x)=L^{-\frac{1}{2}} \phi\left(L^{-2} t, L^{-1} x\right)
$$

and

$$
(\mathcal{S} v)(\phi)=L \mathfrak{s}^{-1} v(\mathfrak{s} \phi)
$$

so that the RG iteration (22) becomes

$$
w_{n-1}^{(N)}(\phi)=\mathcal{S} w_{n}^{(N)}\left(\phi+\Upsilon_{n} *\left(w_{n-1}^{(N)}(\phi)+\xi_{n-1}\right)\right)
$$

where

$$
\Upsilon_{n}=\partial_{x} \Gamma_{n}
$$

Eq. (25) in turn becomes

$$
f_{n-1}^{(N)}(\phi)=L^{-1} \mathcal{S} f_{n}^{(N)}\left(\phi+\Upsilon_{n} *\left(w_{n-1}^{(N)}(\phi)+\xi_{n-1}\right)\right)
$$


and we have the initial conditions

$$
\begin{aligned}
w_{N}^{(N)}(\phi) & =L^{-\frac{1}{2} N}(\phi, M \phi)-L^{-\frac{3}{2} N} C_{L^{-N}} \\
f_{N}^{(N)}(\phi) & =\phi .
\end{aligned}
$$

From now on to avoid too many indices we suppress in the notation the superscript $(N)$ so that $N$ is considered fixed and the scale $n$ runs down from $n=N$.

4.1. Solving the first order. It is instructive and useful to study the fixed point equation (28) to first order in $w$. Define the map

$$
(\mathcal{L} w)(\phi):=\mathcal{S} w\left(\phi+\Upsilon_{n} * \xi_{n-1}\right) .
$$

Then (28) can be written as

$$
w_{n-1}(\phi)=\left(\mathcal{L} w_{n}\right)\left(\phi+\Upsilon_{n} * w_{n-1}(\phi)\right)
$$

so $\mathcal{L}$ is the linearization of the RG map $\mathcal{R}: \mathcal{L}=D \mathcal{R}$. Its properties are crucial for understanding the flow of effective equations $w_{n}$.

Let us consider the linear RG flow from scale $N$ to scale $n$ i.e. $\mathcal{L}^{N-n} w_{N}$. This can be computed by doing one RG step with $L$ replaced by $L^{N-n}$. We get

$$
\mathcal{L}^{N-n} w_{N}(\phi)=\mathcal{S}^{N-n} w_{N}\left(\phi+Y_{n}^{(N)} * \xi_{n}\right)
$$

where

$$
Y_{n}^{(N)}(t, x)=\partial_{x} H_{n}(t, x) \chi_{N-n}(t)
$$

with

$$
H_{n}(t, x)=\frac{1}{\sqrt{4 \pi t}} \sum_{i \in \mathbb{Z}} e^{-\frac{\left(x+i L^{n}\right)^{2}}{4 t}}
$$

being the heat kernel on $\mathbb{T}_{n}$ and

$$
\chi_{m}(s):=\chi(s)-\chi\left(L^{2 m} s\right)
$$

a smooth indicator of the interval $\left[L^{-2 m}, 2\right]$. The field

$$
\vartheta_{n}:=Y_{n}^{(N)} * \xi_{n}
$$

is a stationary Gaussian vector-valued field with covariance given by

$$
\mathbb{E} \vartheta_{n, \alpha}(t, x) \vartheta_{n, \beta}(s, y)=\delta_{\alpha \beta} \mathfrak{C}_{n}^{(N)}(|t-s|, x-y)
$$

where

$$
\mathfrak{C}_{n}^{(N)}(t, x)=-\Delta \int_{0}^{\infty} H_{n}(t+2 \tau, x) \chi_{N-n}(t+\tau) \chi_{N-n}(\tau) d \tau .
$$

The scaling operator has eigenfunctions

$$
\mathcal{S} \phi^{k}=L^{\frac{3-k}{2}} \phi^{k} .
$$

From this one obtains

$$
\mathcal{L}^{N-n} w_{N}(\phi)=L^{-\frac{1}{2} n}\left(\phi+\vartheta_{n}, M\left(\phi+\vartheta_{n}\right)\right)-L^{-\frac{3}{2} n} C_{L^{-N}} .
$$

We see now why the counter term $C_{L^{-N}}$ is needed: the expectation of the random field $\left(\vartheta_{n}, M \vartheta_{n}\right)$ blows up as $N \rightarrow \infty$ as shown in Lemma 4 and this divergence is the source of the renormalization constant $m_{1}$ in (9).

Furthermore, we need to study the dependence of our constructions on the choise of the cutoff function $\chi$ in (7). To this end, let us define

$$
\chi_{m}^{\prime}(s)=\chi(s)-\chi^{\prime}\left(L^{2 m} s\right)
$$


where the lower cutoff in (36) has been replaced by a different bump function $\chi^{\prime}$. In the following we will denote by $Y_{n}^{\prime(N)}$ the kernel $Y_{n}^{(N)}$ where $\chi_{N-n}$ is replaced by $\chi_{N-n}^{\prime}$. We also note that, by taking $\chi^{\prime}(s)=\chi\left(L^{2} s\right)$, one gets $Y_{n}^{\prime(N)}=Y_{n}^{(N+1)}$, so by varying $\chi^{\prime}$ we can also study the dependence and convergence as $N \rightarrow \infty$.

We are now ready to state the Lemma which controls the dependence of the covariance $\mathfrak{C}_{n}^{(N)}$ on $N$ and $\chi$. See the Appendix for the proof.

Lemma 4. Define $m_{1} \in \mathbb{R}^{3}$ by

$$
m_{1}^{(\alpha)}:=\left(\sum_{\beta=1}^{3} M_{\beta \beta}^{(\alpha)}\right) \frac{1}{2^{7 / 2} \sqrt{\pi}} \int_{0}^{\infty} s^{-3 / 2}\left(1-\chi(s)^{2}\right) d s
$$

for $\alpha=1,2,3$. Then

$$
\mathbb{E}\left(\vartheta_{n}^{(N)}, M \vartheta_{n}^{(N)}\right)=L^{N-n} m_{1}+\delta_{n}^{(N)}
$$

where $\left\|\delta_{n}^{(N)}\right\|$ is uniformly bounded in $N$ and $n$ where $\|\cdot\|$ is the Euclidean norm in $\mathbb{R}^{3}$. Moreover, let $\delta_{n}^{\prime(N)}$ be the analog of $\delta_{n}^{(N)}$, where the lower cutoff function is replaced by $\chi^{\prime}$. Then

$$
\left\|\delta_{n}^{(N)}-\delta_{n}^{\prime(N)}\right\| \leq C e^{-c L^{2 N}}\left\|\chi-\chi^{\prime}\right\|_{\infty} .
$$

The counter term $C_{L^{-N}}$ is then given in this linear approximation as

$$
C_{L^{-N}}=L^{N} m_{1}
$$

and we end up with

$$
\mathcal{L}^{N-n} w_{N}(\phi)=u_{n, 1}(\phi)
$$

where

$$
u_{n, 1}(\phi)=L^{-\frac{n}{2}}\left(\left(\phi+\vartheta_{n}, M\left(\phi+\vartheta_{n}\right)\right)-L^{N-n} m_{1}\right) .
$$

4.2. Higher order terms. The heuristic idea of our proof is now the following. We look for the RG flow in the form

$$
w_{n}=\sum_{i=1}^{k-1} u_{n, i}(\phi)+r_{n}
$$

where $u_{n, i}$ are explicit perturbative contributions and in a suitable norm

$$
\left\|u_{n, i}\right\|=\mathcal{O}\left(L^{-\frac{i}{2} n}\right), \quad\left\|r_{n}\right\|=\mathcal{O}\left(L^{-\frac{k}{2} n}\right)
$$

and we expect

$$
r_{n-1}=\mathcal{L} r_{n}+\mathcal{O}\left(L^{-\frac{k+1}{2} n}\right) .
$$

Moreover, from our analysis of $\mathcal{L}$ we also expect that

$$
\left\|\mathcal{L} r_{n}\right\| \leq C L^{\frac{3}{2}}\left\|\mathcal{L} r_{n}\right\| \leq C L^{\frac{3}{2}} L^{-\frac{k}{2} n}=C L^{\frac{3}{2}-\frac{k}{2}} L^{-\frac{k}{2}(n-1)}
$$

so that (49) should iterate provided we take $k=4$. Hence, we should find the perturbative contributions to $w_{n}$ up to order 3.

Remark 5. The same heuristic idea works in general for subcritical problems. The dimensionless strength of the nonlinearity is $L^{-N \alpha}$ for some $\alpha>0$ and the norm of $\mathcal{L}$ is $L^{\beta}$ for some $\beta>0$. Then one needs to do perturbation theory up to order $k-1$ with $k \alpha>\beta$. 
The $u_{n, i}$ may be computed by doing one RG step with scaling factor $L^{N-n}$

$$
w_{n}(\phi)=L^{-\frac{n}{2}}\left(\phi+\vartheta_{n}+Y_{n} * w_{n}(\phi), M\left(\phi+\vartheta_{n}+Y_{n} * w_{n}(\phi)\right)\right)
$$

where we dropped the superscript $N$ also in $Y_{n}^{(N)}$. We obtain

$$
u_{n, 2}(\phi)=2 L^{-n}\left(\phi+\vartheta_{n}, M\left(Y_{n} * u_{n, 1}(\phi)\right)\right.
$$

and

$$
\begin{aligned}
u_{n, 3}(\phi)= & L^{-\frac{3}{2} n}\left(\left(Y_{n} * u_{n, 1}(\phi), M\left(Y_{n} * u_{n, 1}(\phi)\right)\right.\right. \\
& +2\left(\phi+\vartheta_{n}, M\left(Y_{n} * u_{n, 2}(\phi)\right)-m_{2} \log L^{N}-m_{3}\right)
\end{aligned}
$$

where $m_{2}$ and $m_{3}$ are constants to be determined. To write the recursion (49) let us denote $w_{n}$ by $w$ and $w_{n-1}$ by $w^{\prime}$ and similarly for the other functions. Then

$$
r^{\prime}(\phi)=\mathcal{L} r\left(\phi+\Upsilon * w^{\prime}(\phi)\right)+\mathcal{F}\left(r^{\prime}\right)(\phi)
$$

with

$$
\begin{aligned}
\mathcal{F}\left(r^{\prime}\right)(\phi)= & \left.u_{1}^{\prime}\left(\phi+\Upsilon * w^{\prime}\right)-u_{1}^{\prime}(\phi)-D u_{1}^{\prime}(\phi)\left(\Upsilon *\left(u_{1}^{\prime}+u_{2}^{\prime}\right)\right)-\frac{1}{2} D^{2} u_{1}^{\prime}(\phi)\left(\Upsilon * u_{1}^{\prime}, \Upsilon * u_{1}^{\prime}\right)\right) \\
& +\mathcal{L} u_{2}\left(\phi+\Upsilon * w^{\prime}\right)-\mathcal{L} u_{2}(\phi)-D \mathcal{L} u_{2}(\phi) \Upsilon * u_{1}^{\prime} \\
& +\mathcal{L} u_{3}\left(\phi+\Upsilon * w^{\prime}\right)-\mathcal{L} u_{3}(\phi) \\
\equiv & \mathcal{F}_{1}\left(r^{\prime}\right)(\phi)+\mathcal{F}_{2}\left(r^{\prime}\right)(\phi)+\mathcal{F}_{3}\left(r^{\prime}\right)(\phi)
\end{aligned}
$$

where $D$ is the (Frechet) derivative and on the LHS $w^{\prime}, u^{\prime}$ are evaluated at $\phi$.

Remark 6. Note that $u_{i}$ are polynomials in $\phi$ so there is no problem in defining the derivative. In Section 6 we'll see that $w$ is actually analytic.

\section{RANDOM FIELDS}

The perturbative terms $u_{i}$ are polynomials in $\phi$ with random coefficients. For the heuristic idea of the proof presented above to work these coefficients should not be too large. For $u_{n, 1}$ these random coefficients are the random fields $\vartheta_{n}(t, x)$ and

$$
u_{n, 1}(0)=L^{-\frac{n}{2}}\left(\left(\vartheta_{n}(t, x), M \vartheta_{n}(t, x)\right)-L^{N-n} m_{1}\right)
$$

In case of $u_{n, 2}$ and $u_{n, 3}$ we don't need to consider all the coefficients. Indeed, the discussion of previous section was based on a bound $L^{\frac{3}{2}}$ for the linearized RG operator. This is indeed its eigenvalue on constants. The next eigenvalue is $L$ on linear functions, the one after $L^{\frac{1}{2}}$ etc. Thus for $u_{n, 2}$ we should be worried only about the constant and linear terms in $\phi$ and for $u_{n, 3}$ only about constants. All the other terms should be irrelevant i.e. they should contract under the RG. We will now isolate these relevant terms. Let us expand

$$
u_{n, 2}(\phi)=u_{n, 2}(0)+D u_{n, 2}(0) \phi+U_{n, 2}(\phi) .
$$

We get

and

$$
u_{n, 2}(0)=2 L^{-n}\left(\vartheta_{n}, M Y_{n} * u_{n, 1}(0)\right)
$$

$$
D u_{n, 2}(0) \phi=L^{-n}\left(\phi, M Y_{n} * u_{n, 1}(0)\right)+L^{-n} \int_{-\infty}^{t} d s \int_{\mathbb{T}_{n}} d y \sigma_{n}(t, x, s, y) \phi(s, y)
$$


where

$$
\left.\left(\sigma_{n}(t, x, s, y)\right)_{\alpha \beta}=4 Y_{n}(t-s, x-y) \sum_{\gamma, \delta, \lambda} \vartheta_{\gamma}(t, x) M_{\gamma \delta}^{(\alpha)} \vartheta_{\lambda}(s, y)\right) M_{\lambda \beta}^{(\delta)} .
$$

For the third order term we get

$$
u_{n, 3}(\phi)=u_{n, 3}(0)+U_{n, 3}(\phi) .
$$

with

$$
\begin{aligned}
u_{n, 3}(0)= & L^{-\frac{3}{2} n}\left[\left(Y_{n} * u_{n, 1}(0), M\left(Y_{n} * u_{n, 1}(0)\right)\right.\right. \\
& +\left(\vartheta_{n}, M\left(Y_{n} * u_{n, 2}(0)\right)-m_{2} \log L^{N}-m_{3}\right] .
\end{aligned}
$$

Consider now the random fields $u_{n, i}(0), D u_{n, 2}(0)$ with the scaling factor divided out, i.e.

$$
\mathfrak{z}_{n, i}:=L^{\frac{i}{2} n} u_{n, i}(0), \quad D \mathfrak{z}_{n, 2}:=L^{n} D u_{n, 2}(0) .
$$

Then $\vartheta_{n}, \mathfrak{z}_{n, i}, D \mathfrak{z}_{n, 2}$ belong to the Wiener chaos of white noise of bounded order $(\leq 4)$ and their size and regularity are controlled by studying their covariances, as shown in the Section 8 . For finite cutoff parameter $N$ these noise fields are a.s. smooth but in the limit $N \rightarrow \infty$ they become distribution valued. We estimate their size in suitable (negative index) Sobolev type norms which we now define.

The operator $\left(-\partial_{t}^{2}+1\right)^{-1}$ acts on $L^{2}(\mathbb{R})$ by convolution with the function

$$
K_{1}(t)=\frac{1}{2} e^{-|t|}
$$

and the operator $(-\Delta+1)^{-1}$ on $L^{2}\left(\mathbb{T}_{n}\right)$ is convolution with the periodization of (62)

$$
K_{2}(x)=\sum_{i \in \mathbb{Z}} K_{1}\left(x+i L^{n}\right)
$$

Let

$$
K(t, x)=K_{1}(t) K_{2}(x) .
$$

Note that convolution with $K$ is a positive operator in $L^{2}\left(\mathbb{R} \times \mathbb{T}_{n}\right)$. We define $\mathcal{V}_{n}$ to be be the completion of $C_{0}^{\infty}\left(\mathbb{R}_{+} \times \mathbb{T}_{n}\right)$ with the norm

$$
\|v\|_{\mathcal{V}_{n}}=\sup _{i}\|K * v\|_{L^{2}\left(\mathfrak{c}_{i}\right)}
$$

where $\mathfrak{c}_{i}$ is the unit cube centered at $i \in \mathbb{Z} \times\left(\mathbb{Z} \cap \mathbb{T}_{n}\right)$. To deal with the bi-local field as $\sigma_{n}$ in (58) we define for $\sigma(t, x, s, y)$ in $C_{0}^{\infty}\left(\mathbb{R}_{+} \times \mathbb{T}_{n} \times \mathbb{R}_{+} \times \mathbb{T}_{n}\right)$

$$
\|\sigma\| \mathcal{V}_{n}=\sup _{i} \sum_{j}\|K \otimes K * \sigma\|_{L^{2}\left(\mathfrak{c}_{i} \times \mathfrak{c}_{j}\right)}
$$

Now we can specify the admissible set of noise. Let $\gamma>0$ and define the sets of events $\mathcal{E}_{m}, m>0$ in the probability space of the space time white noise $\Xi$ as follows. Let $\zeta_{n}^{(N)}$ denote any fields $\vartheta_{n}, \mathfrak{z}_{n, i}, D_{\mathfrak{z} n, 2}$. The first condition on $\mathcal{E}_{m}$ is that for all $N \geq n \geq m$ the following hold:

$$
\left\|h_{n} \zeta_{n}^{(N)}\right\|_{\mathcal{V}_{n}} \leq L^{\gamma n}
$$

where $h_{n}$ is a smooth indicator of the time interval $\left[0, \tau_{n}\right], \tau_{n}=L^{2(n-m)}$ which is introduced to localize in time the flow equation, as we will see in Section [6. More precisely, $h$ is a smooth bump on $\mathbb{R}$ with $h(t)=1$ for $t \leq-L^{-2}$ and $h(t)=0$ for $t \geq-\frac{1}{2} L^{-2}$ and set $h_{k}(t)=h\left(t-\tau_{k}\right)$ so that $h_{k}(t)=1$ for $t \leq \tau_{k}-L^{-2}$ and $h_{k}(t)=0$ for $t \geq \tau_{k}-\frac{1}{2} L^{-2}$. 
We need also to control the $N$ and $\chi$ dependence of the noise fields $\zeta_{n}^{(N)}$. We can study both by varying the lower cutoff in the operator $Y_{n}^{(N)}$ in (34). We denote by $\zeta_{n}^{\prime(N)}$ any of the resulting noise fields. Our second condition on $\mathcal{E}_{m}$ is that for all $N \geq n \geq m$ and all cutoff functions $\chi, \chi^{\prime}$ with bounded $C^{1}$ norm

$$
\left\|h_{n}\left(\zeta_{n}^{\prime(N)}-\zeta_{n}^{(N)}\right)\right\| \mathcal{V}_{n} \leq L^{-\gamma(N-n)} L^{\gamma n}
$$

The final condition concerns the fields $\Upsilon_{n} * \xi_{n-1}$ entering the RG iteration (28). Note that these fields are $N$ independent and smooth and we are going to impose on them a smoothness condition: for all $n>m$ we demand

$$
\left\|\Upsilon_{n} * \xi_{n-1}\right\|_{\Phi_{n-1}} \leq L^{\gamma n}
$$

where the norm is defined in next section. In Section 8 we prove

Proposition 7. There exists $\gamma>0$ such that almost surely $\mathcal{E}_{m}$ holds for some $m<\infty$.

In the following sections we suppose the noise is on $\mathcal{E}_{m}$ and we will control the RG iteration (51) for scales $n \geq m$.

\section{BANACH SPACE SETUP FOR THE RG MAP}

In this section we set up the RG iteration in suitable functional spaces along the same lines of [10, 11]. Let us first discuss the domain and range of the effective nonlinearities $w_{n}$. The range of $w_{n}, r_{n}$ is dictated by the noise, so we take it to be $\mathcal{V}_{n}$.

In the argument of $w_{n}$ in (28) $\Upsilon_{n} *\left(w_{n-1}+\xi_{n-1}\right)$ is smooth so we take the domain of $w_{n}(\phi)$ to consist of suitably smooth functions. Let $\Phi_{n}$ be the space of

$$
\phi:\left[0, \tau_{n}\right] \times \mathbb{T}_{n} \rightarrow \mathbb{C}
$$

which are $C^{2}$ in $t$ and $C^{2}$ in $x$ with $\partial_{t}^{i} \phi(0, x)=0$ for $0 \leq i \leq 2$ and all $x \in \mathbb{T}_{n}$. We equip $\Phi_{n}$ with the sup norm

$$
\|\phi\|_{\Phi_{n}}:=\sum_{i \leq 2, j \leq 2}\left\|\partial_{t}^{i} \partial_{x}^{j} \phi\right\|_{\infty} .
$$

The following lemma collects some elementary facts on how our spaces tie up with the operators entering the RG:

Lemma 8. (a) $\Upsilon_{n}: \mathcal{V}_{n-1} \rightarrow \Phi_{n-1}$ and $h_{n-1} \Upsilon_{n}: \mathcal{V}_{n-1} \rightarrow \mathcal{V}_{n-1}$ are bounded operator with norm $C(L)$.

(b) $\mathfrak{s}: \Phi_{n-1} \rightarrow \Phi_{n}$ and $\mathfrak{s}^{-1}: \mathcal{V}_{n} \rightarrow \mathcal{V}_{n-1}$ are bounded with

$$
\|\mathfrak{s}\| \leq L^{-\frac{1}{2}}, \quad\left\|\mathfrak{s}^{-1}\right\| \leq C L^{\frac{1}{2}}
$$

(c) Let $\phi \in C^{2,2}\left(\mathbb{R} \times \mathbb{T}_{n}\right)$ and $v \in \mathcal{V}_{n}$. Then $\phi v \in \mathcal{V}_{n}$ and $\|\phi v\| \mathcal{V}_{n} \leq C\|\phi\|_{C^{2,2}}\|v\|_{\mathcal{V}_{n}}$.

Proof. Essentially the same as Lemma 9 in [5].

Consider now our fixed point problem

$$
w_{n-1}(\phi)=\mathcal{S} w_{n}\left(\phi+\Upsilon_{n} * \xi_{n-1}+\Upsilon_{n} * w_{n-1}(\phi)\right)
$$

$w_{n}$ takes values in the distribution space $\mathcal{V}_{n} \subset \mathcal{D}^{\prime}\left(\mathbb{R}_{+} \times \mathbb{T}_{n}\right)$. We want to bound it on the time interval $\left[0, \tau_{n}\right]$ i.e. we need to localize (68) in time. Define

$$
\tilde{w}_{n}=h_{n} w_{n}
$$

so that

$$
\tilde{w}_{n-1}(\phi)=h_{n-1} \mathcal{S} w_{n}\left(\phi+\Upsilon_{n} * \xi_{n-1}+\Upsilon_{n} * w_{n-1}(\phi)\right)
$$


One can readily check that $\Upsilon_{n} w_{n-1}=\Upsilon_{n} \tilde{w}_{n-1}$ on the time interval $\left[0, \tau_{n-1}\right]$ and that

$$
h_{n-1} \mathcal{S} w_{n}=h_{n-1} \mathcal{S} \tilde{w}_{n} .
$$

Thus (69) can be written as

$$
\tilde{w}_{n-1}(\phi)=h_{n-1} \mathcal{S} \tilde{w}_{n}\left(\phi+\Upsilon_{n} * \xi_{n-1}+\Upsilon_{n} * \tilde{w}_{n-1}(\phi)\right) .
$$

We will solve (70) in a space of analytic functions which we discuss next. Let $\mathcal{H}, \mathcal{H}^{\prime}$ be Banach spaces and $B(r) \subset \mathcal{H}$ open ball of radius $r$. Let $H^{\infty}\left(B(r), \mathcal{H}^{\prime}\right)$ denotes the space of analytic functions $f: B(r) \rightarrow \mathcal{H}^{\prime}$ with sup norm which we denote by $\||\cdot|\|_{B(r)}$. We will use the following simple facts that are identical to those of analytic functions on finite dimensional spaces (see [9]).

(a). Let $w \in H^{\infty}\left(B(r), \mathcal{H}^{\prime}\right)$ and $w^{\prime} \in H^{\infty}\left(B\left(r^{\prime}\right), \mathcal{H}^{\prime \prime}\right)$. If $\||w|\|<r^{\prime}$ then $w^{\prime} \circ w \in$ $H^{\infty}\left(B(r), \mathcal{H}^{\prime \prime}\right)$ and

$$
\left\|\left|w^{\prime} \circ w\left\|_{B(r)} \leq\right\|\right|\left|w^{\prime}\right|\right\|_{B\left(r^{\prime}\right)} .
$$

(b). Let $w \in H^{\infty}\left(B(r), \mathcal{H}^{\prime}\right)$ and $\rho<r$. Then

$$
\sup _{\|x\|<\rho}\|D w(x)\|_{\mathcal{L}\left(\mathcal{H}, \mathcal{H}^{\prime}\right)} \leq(r-\rho)^{-1}\|\| w \|_{B(r)},
$$

where $\mathcal{L}\left(\mathcal{H}, \mathcal{H}^{\prime}\right)$ denotes the space of bounded linear operators from $\mathcal{H}$ to $\mathcal{H}^{\prime}$. Taking $\rho=\frac{1}{2} r^{\prime}$, we infer that if $\left\|\mid w_{i}\right\|_{B(r)} \leq \frac{1}{2} \rho$ then

$$
\left\|\left|w^{\prime} \circ w_{1}-w^{\prime} \circ w_{2}\right|\right\|_{B(r)} \leq \frac{2}{r^{\prime}}|| w^{\prime}||_{B\left(r^{\prime}\right)}||\left|w_{1}-w_{2}\right| \|_{B(r)} .
$$

(c). Define $\delta_{k} w(x):=w(x)-\sum_{\ell=0}^{k-1} \frac{1}{\ell !} D^{\ell} w(0)(x)$. Then

$$
\left\|\left|\delta_{k} w\right|\right\|_{B(a r)} \leq \frac{a^{k}}{1-a}|||w| \|_{B(r)}
$$

for $0 \leq a<1$.

Furthermore, we infer this important corollary from Lemma 8

Proposition 9. $\mathcal{S}$ maps $H^{\infty}\left(B(R), \mathcal{V}_{n}\right)$ into $H^{\infty}\left(B\left(L^{\frac{1}{2}} R\right), \mathcal{V}_{n-1}\right)$ with norm $\|\mathcal{S}\| \leq$ $C L^{\frac{3}{2}}$. Here $B(R) \subset \Phi_{n}$ and $B\left(L^{\frac{1}{2}} R\right) \subset \Phi_{n-1}$ respectively.

Let now $\gamma>0$ and set $B_{n}=B\left(L^{2 \gamma n}\right) \subset \Phi_{n}$. Then we have

Proposition 10. There exist $L_{0}>0, \gamma_{0}>0$ so that for $L>L_{0}, \gamma<\gamma_{0}$ and $m>$ $m(\gamma, L)$ if $\Xi \in \mathcal{A}_{m}$ then then for all $N \geq n-1 \geq m$ the equation (70) has a unique solution $\tilde{w}_{n-1}^{(N)} \in H^{\infty}\left(B_{n-1}, \mathcal{V}_{n-1}\right)$. These solutions satisfy

$$
\left\|\tilde{w}_{n}^{(N)}\right\| \|_{B_{n}} \leq L^{-\frac{1}{4} n}
$$

and $\tilde{w}_{n}^{(N)}$ converge in $H^{\infty}\left(B_{n}, \mathcal{V}_{n}\right)$ to a limit $\tilde{w}_{n}$ as $N \rightarrow \infty$. Furthermore, $\tilde{w}_{n}$ is independent on the small scale cutoff.

Proof. We will drop the tilde from now on so that $w_{n}, r_{n}$ and $u_{n, i}$ stand for $\tilde{w}_{n}$ etc. Also, if no confusion arises we let $w$ and $w^{\prime}$ stand for $w_{n}$ and $w_{n-1}$ respectively. We 
start with the perturbative contributions $u_{i}$. As a corollary of Lemma 8 (c) and (65) we obtain for $n \geq m$ and $N \geq n$ :

$$
\begin{aligned}
\left\|u_{n, 1}\right\|_{R B_{n}} & \leq C R^{2} L^{\left(4 \gamma-\frac{1}{2}\right) n} \\
\left\|\mid u_{n, 2}(0)+D u_{n, 2}(0) \phi\right\|_{R B_{n}} & \leq C R L^{(3 \gamma-1) n} \\
\left\|u_{n, 3}(0)\right\| \mathcal{V}_{n} & \leq C L^{\left(\gamma-\frac{3}{2}\right) n}
\end{aligned}
$$

for all $R \geq 1$. We used also $\left\|h_{n}\right\|_{C^{2,2}} \leq C$.

We need to bound the remainder terms $U_{2}$ and $U_{3}$ in (55) and (59). We do this inductively in $n$. We have

$$
u_{2}^{\prime}(\phi)=\mathcal{L} u_{2}(\phi)+D u_{1}^{\prime}(\phi) \Upsilon_{n} u_{1}^{\prime}(\phi):=\mathcal{L} u_{2}(\phi)+v_{2}(\phi)
$$

Using Lemma 8(a), (76) and (72) we get

$$
\left\|\left|v_{2}\right|\right\|_{L^{\frac{1}{2}} B_{n-1}} \leq C(L) L^{(6 \gamma-1) n} .
$$

Let us inductively assume

$$
\left.||\left|u_{n, 2}\right|\right|_{B_{n}} \leq C L^{(7 \gamma-1) n} .
$$

Using Proposition 9 and (67) we get the following useful result

$$
\left\|\left|\mathcal{L} W\left\|_{L^{\frac{1}{2}} B_{n-1}} \leq C L^{\frac{3}{2}}\right\|\right| W\right\|_{B_{n}} .
$$

for all $W \in H^{\infty}\left(B_{n}, \mathcal{V}_{n}\right)$ since $B\left(L^{2 \gamma(n-1)}+L^{\gamma n}\right) \subset B_{n}$ if $L>L(\gamma)$. Thus

$$
\left\|\left|u_{2}^{\prime}\right|\right\|_{L^{\frac{1}{2}} B_{n-1}} \leq C L^{\frac{3}{2}}||\left|u_{2}\right| \| B_{B_{n}}+C(L) L^{(6 \gamma-1) n} \leq C L^{\frac{3}{2}} L^{(7 \gamma-1) n}
$$

if $n>n(\gamma, L)$. Then by (74)

$$
\text { ||| } U_{2}^{\prime}||_{B_{n-1}}=||\left|\delta_{2} u_{2}^{\prime}\right| \|_{B_{n-1}} \leq C L^{\frac{1}{2}} L^{(7 \gamma-1) n} .
$$

Using (77), the bound (79) follows for $n-1$ provided we take $\gamma$ so that $\frac{1}{2}+(7 \gamma-1)<0$. For $u_{n, 3}$ we have the recursion

$$
u_{3}^{\prime}(\phi)=\mathcal{L} u_{3}(\phi)+v_{3}(\phi)
$$

with

$$
v_{3}(\phi)=\frac{1}{2} D^{2} u_{1}^{\prime}(\phi)\left(\Upsilon u_{1}^{\prime}, \Upsilon u_{1}^{\prime}\right)+D u_{1}^{\prime}(\phi) \Upsilon u_{2}^{\prime}+D \mathcal{L} u_{2}(\phi) \Upsilon u_{1}^{\prime} .
$$

We readily get

The inductive bound

$$
\left\|\left|v_{3}\right|\right\|_{L^{\frac{1}{2}} B_{n-1}} \leq C(L) L^{\left(8 \gamma-\frac{3}{2}\right) n} .
$$

$$
\left\|\mid u_{n, 3}\right\|_{B_{n}} \leq C L^{\left(9 \gamma-\frac{3}{2}\right) n} .
$$

follows then in the same way as for $u_{2}$, using $U_{3}=\delta_{1} u_{3}$.

Now we are ready to solve equation (51) by Banach fixed point theorem. Thus consider the map

$$
\mathcal{G}\left(r^{\prime}\right)=\mathcal{L} r\left(\phi+\Upsilon * w^{\prime}\right)+\mathcal{F}\left(r^{\prime}\right)
$$

where $\mathcal{F}\left(r^{\prime}\right)$ is given by (53).

We have

$\mathcal{F}_{1}\left(r^{\prime}\right)(\phi)=L^{-\frac{n-1}{2}}\left(2\left(\phi+\vartheta, M\left(\Upsilon *\left(u_{3}^{\prime}+r^{\prime}\right)\right)+\left(\Upsilon *\left(u_{2}^{\prime}+u_{3}^{\prime}+r^{\prime}\right), M \Upsilon *\left(u_{2}^{\prime}+u_{3}^{\prime}+r^{\prime}\right)\right)\right)\right.$ so that

$$
\||| \mathcal{F}_{1}\left(r^{\prime}\right)||_{B_{n-1}} \leq C(L)\left(L^{(14 \gamma-2)(n-1)}+L^{\left(2 \gamma-\frac{1}{2}\right)(n-1)}\left\||| r^{\prime} \mid\right\|_{B_{n-1}}\right) .
$$


Next we write

$$
\begin{aligned}
\mathcal{F}_{2}\left(r^{\prime}\right) & =\mathcal{L} u_{2}\left(\phi+\Upsilon * w^{\prime}\right)-\mathcal{L} u_{2}\left(\phi+\Upsilon * u_{1}^{\prime}\right) \\
& +\mathcal{L} u_{2}\left(\phi+\Upsilon * u_{1}^{\prime}\right)-\mathcal{L} u_{2}(\phi)-D \mathcal{L} u_{2}(\phi) \Upsilon * u_{1}^{\prime} \\
& \equiv \mathcal{F}_{2,1}\left(r^{\prime}\right)+\mathcal{F}_{2,2}\left(r^{\prime}\right)
\end{aligned}
$$

Using (73) we obtain

$$
\left\|\left|\mathcal{F}_{2,1}\left(r^{\prime}\right)\right|\right\|_{B_{n-1}} \leq C(L)\left(L^{(14 \gamma-2)(n-1)}+L^{(7 \gamma-1)(n-1)}\left|\left\|r^{\prime} \mid\right\|_{B_{n-1}}\right)\right.
$$

To bound $\mathcal{F}_{2,2}\left(r^{\prime}\right)$ consider the function $f(z)=\mathcal{L} u_{2}\left(\phi+z \Upsilon * u_{1}^{\prime}\right)$ for $z \in \mathbb{C}$. Since $\mathcal{L} u_{2}$ is analytic in $L^{\frac{1}{2}} B_{n-1}$ and

$$
\left\|\phi+z \Upsilon * u_{1}^{\prime}\right\|_{\Phi_{n-1}} \leq L^{2 \gamma(n-1)}+C L^{\left(4 \gamma-\frac{1}{2}\right)(n-1)}|z|
$$

we get that $f$ is analytic in the ball $|z| \leq C L^{\left(\frac{1}{2}-2 \gamma\right)(n-1)}$. Since $\mathcal{F}_{2,2}\left(r^{\prime}\right)(\phi)=f(1)-$ $f(0)-f^{\prime}(0)$ we conclude by a Cauchy estimate

$$
\left\|\mathcal{F}_{2,2}\left(r^{\prime}\right) \mid\right\|_{B_{n-1}} \leq C(L) L^{(15 \gamma-2)(n-1)} .
$$

For $\mathcal{F}_{3}$ we get using (80) and (73)

$$
\|\left.\left|\mathcal{F}_{3}\left(r^{\prime}\right)\right|\right|_{B_{n-1}} \leq C(L)\left(L^{(13 \gamma-2)(n-1)}+L^{(9 \gamma-2)(n-1)}||\left|r^{\prime}\right| \|_{B_{n-1}}\right)
$$

Consider finally the first term in (83). (80) implies

$$
\left\||| \mathcal{L} r\left(\cdot+\Upsilon * w^{\prime}\right)||_{B_{n-1}} \leq C L^{\frac{3}{2}} \mid\right\| r \|_{B_{n}} .
$$

We conclude that by taking $\gamma$ small enough if

$$
\||| r \mid\|_{B_{n}} \leq L^{-\frac{7}{4} n}
$$

then $\mathcal{G}$ maps the ball $\left\|\left|r^{\prime}\right|\right\|_{B_{n-1}} \leq L^{-\frac{7}{4}(n-1)}$ to itself. It is now straightforward to check that $\mathcal{G}$ is a contraction in this ball so that by induction in $n$ (85) holds for all $n \geq m$.

Let us address the convergence as $N \rightarrow \infty$ and cutoff dependence of $w_{n}=w_{n}^{(N)}$ which can be dealt with together by considering the difference $w_{n}-w_{n}^{\prime}$ where $w_{n}^{\prime}$ equals $w_{n}^{N+1}$ or $w_{n}^{N}$ with a different cutoff. We proceed as with $w_{n}$, starting with the following bounds that follow from (66): for all $n \geq m$ and $N \geq n$

$$
\begin{aligned}
\left\|u_{n, 1}-u_{n, 1}^{\prime} \mid\right\|_{R B_{n}} & \leq C R^{2} L^{-\gamma(N-n)} L^{\left(4 \gamma-\frac{1}{2}\right) n} \\
\left\|u_{n, 2}(0)+D u_{n, 2}(0) \phi-\left(u_{n, 2}^{\prime}(0)+D u_{n, 2}^{\prime}(0) \phi\right)\right\| \|_{R B_{n}} & \leq C R L^{-\gamma(N-n)} L^{(3 \gamma-1) n} \\
\left\|u_{n, 3}(0)-u_{n, 3}^{\prime}(0)\right\|_{\mathcal{V}_{n}} & \leq C L^{-\gamma(N-n)} L^{\left(\gamma-\frac{3}{2}\right) n}
\end{aligned}
$$

for all $R \geq 1$. The induction then goes as for $w_{n}^{N}$, except for the prefactor $L^{-\gamma(N-n)}$ in all the bounds. This establishes the convergence of $w_{n}^{(N)}$ to a limit that is independent on the short time cutoff.

\section{Proof of Theorem 1}

We can now construct the solution $\varphi^{(\epsilon)}$ of the $\epsilon$ cutoff equation (111) and consequently $u^{(\epsilon)}$ in (6). Recall that formally $u^{(\epsilon)}$ is given on time interval $\left[0, L^{-2 m}\right]$ by equation (27) with $n=m$ and $\varphi_{m}$ is the solution of equation (23) on time interval $[0,1]$. Hence we first need to study the $f$ iteration equation (25) which is equivalent to (29). We study instead of (29) the localized iteration

$$
\tilde{f}_{n-1}^{(N)}(\phi)=h_{n-1} L^{-1} \mathcal{S} \tilde{f}_{n}^{(N)}\left(\phi+\Upsilon_{n} *\left(\tilde{w}_{n-1}^{(N)}(\phi)+\xi_{n-1}\right)\right)
$$


for $\tilde{f}_{n}^{(N)}=h_{n} f_{n}^{(N)}$. Then we can show the following Proposition.

Proposition 11. Let $\tilde{w}_{n}^{(N)} \in H^{\infty}\left(B_{n}, \mathcal{V}_{n}\right), m \leq n \leq N$ be as in Proposition 10 and $\phi \in$ $B_{n}$. Then for $m \leq n \leq N \tilde{f}_{n}^{(N)} \in H^{\infty}\left(B_{n}, \mathcal{V}_{n}\right)$ and $\tilde{f}_{n}^{(N)}(\phi)$ converges in $H^{\infty}\left(B_{n}, \mathcal{V}_{n}\right)$ as $N \rightarrow \infty$ to a limit $\psi_{n}$ which is independent of the cutoff function.

Proof. Let us write

$$
\tilde{f}_{n}^{(N)}(\phi)=h_{n}\left(\phi+\vartheta_{n}^{(N)}\right)+g_{n}^{(N)}(\phi)
$$

Then

$$
g_{n-1}^{(N)}(\phi)=h_{n-1} L^{-1} \mathcal{S} g_{n}^{(N)}\left(\phi+\Upsilon_{n} *\left(\tilde{w}_{n-1}^{(N)}(\phi)+\xi_{n-1}\right)\right)+h_{n-1} \Upsilon_{n} * \tilde{w}_{n-1}^{(N)}(\phi)
$$

Note that the operator $L^{-1} \mathcal{S}$ has norm bounded by $C L^{\frac{1}{2}}$ and $\tilde{w}_{n}^{(N)}$ has norm bounded by $C L^{\left(-\frac{1}{2}+4 \gamma\right) n}$. Hence we need to extract the leading "marginal" part from $\tilde{w}_{n}^{(N)}$ :

$$
g_{n}^{(N)}=h_{n} Y_{n}^{(N)} * \tilde{u}_{n, 1}^{(N)}+b_{n}^{(N)} .
$$

As we will see in Section 8 (Lemma 14), uniformly in $n$ we have

$$
\mathcal{Y}_{n}:=\sup _{N \geq n}\left|Y_{n}^{(N)}\right| \in L^{p}\left(\mathbb{R} \times \mathbb{T}_{n}\right), \quad\left\|Y_{n}^{(N)}-Y_{n}^{(N)}\right\|_{p}^{p} \leq C L^{-\lambda(N-n)}\left\|\chi^{\prime}-\chi\right\|_{\infty}
$$

for $p<\frac{3}{2}$ and some $\lambda>0$. Then thanks to Lemma 8 (c) and Young's inequality we have

$$
\begin{aligned}
\left\|h_{n} Y_{n}^{(N)} * \tilde{u}_{n, 1}^{(N)}\right\|_{\mathcal{V}_{n}} & \leq C \sum_{i}\left\|K * Y_{n}^{(N)} * \tilde{u}_{n, 1}\right\|_{L^{2}\left(\mathfrak{c}_{i}\right)} \leq C \sum_{i}\left\|\mathcal{Y}_{n}\right\|_{L^{1}\left(\mathfrak{c}_{i}\right)}\left\|K * \tilde{u}_{n, 1}\right\|_{L^{2}\left(\mathfrak{c}_{i}\right)} \\
& \leq C\left\|\mathcal{Y}_{n}\right\|_{L^{1}\left(\mathbb{R} \times \mathbb{T}_{n}\right)} \sum_{i}\left\|K * \tilde{u}_{n, 1}\right\|_{L^{2}\left(\mathfrak{c}_{i}\right)} \\
& \leq C\left\|\tilde{u}_{n, 1}\right\| \mathcal{\nu}_{n} .
\end{aligned}
$$

Thus $\left\|\left|h_{n} Y_{n}^{(N)} * \tilde{u}_{n, 1}^{(N)}\right|\right\|_{B_{n}} \leq C L^{\left(4 \gamma-\frac{1}{2}\right) n}$ by Proposition 10. Then the iteration of $b_{n}^{(N)}$ gives easily $\left\||| b_{n}^{(N)}\right\| \|_{B_{n}} \leq L^{-\frac{3}{4} n}$, which implies that $\left\|\mid g_{n}^{(N)}\right\| \|_{B_{n}} \leq L^{-\frac{1}{4} n}$.

The convergence and cutoff independence follows from that of $\tilde{w}_{n}^{(N)}$ proved in Proposition 10 ,

Moreover, we need also this technical Lemma.

Lemma 12. $\partial_{x} G_{1}$ is a bounded operator from $\mathcal{V}_{n}$ to $\Phi_{n}$ and $\partial_{x} G_{1} *\left(h_{n-1}\left(L^{-2} \cdot\right) v\right)=$ $\partial_{x} G_{1} * v$.

Proof. As in [5], Lemma 14.

Now we can finally prove our main result: let $\phi_{n} \in \Phi_{n}$ be defined inductively by $\phi_{m}=0$ and for $n>m$

$$
\phi_{n}=\mathfrak{s}\left(\phi_{n-1}+\Upsilon_{n} *\left(\tilde{w}_{n-1}^{(N)}\left(\phi_{n-1}\right)+\xi_{n-1}\right)\right) .
$$

We claim that for all $m \leq n \leq N \phi_{n} \in B_{n}$ and

$$
\phi_{n}=\partial_{x} G_{1} *\left(\tilde{w}_{n}^{(N)}\left(\phi_{n}\right)+\xi_{n}\right) .
$$

Indeed, this holds trivially for $n=m$ since the RHS vanishes identically on $[0,1]$. Suppose $\phi_{n-1} \in B_{n-1}$ satisfies

$$
\phi_{n-1}=\partial_{x} G_{1} *\left(\tilde{w}_{n-1}^{(N)}\left(\phi_{n-1}\right)+\xi_{n-1}\right) .
$$


Then, first by Lemma 8 (b) and (무)

$$
\left\|\phi_{n}\right\|_{\Phi_{n}} \leq L^{-\frac{1}{2}}\left\|\phi_{n-1}\right\|_{\Phi_{n-1}}+C(L) L^{\gamma n} \leq L^{2 \gamma n}
$$

so that $\phi_{n} \in B_{n}$. Second, we have by (92), (90) and Lemma 12

$$
\begin{aligned}
\phi_{n} & =\mathfrak{s}\left(\left(\partial_{x} G_{1}+\Upsilon\right) *\left(\tilde{w}_{n-1}^{(N)}\left(\phi_{n-1}\right)+\xi_{n-1}\right)\right) \\
& =\partial_{x} G_{1} *\left(\tilde{w}_{n}^{(N)}\left(\phi_{n}\right)+\xi_{n}\right) .
\end{aligned}
$$

Since $\phi_{m}=0$, from (89) we have

$$
\tilde{f}_{m}^{(N)}(0)=h_{m} \mathfrak{s}^{-1} \tilde{f}_{m+1}^{(N)}\left(\phi_{m+1}\right)=h_{m} h_{m+1}\left(L^{2} \cdot\right) \mathfrak{s}^{-2} \tilde{f}_{m+2}^{(N)}\left(\phi_{m+2}\right)=h_{m} \mathfrak{s}^{-2} \tilde{f}_{m+2}^{(N)}\left(\phi_{m+2}\right),
$$

then, iterating we get

$$
\tilde{f}_{m}^{(N)}(0)=h_{m} \mathfrak{s}^{-(N-m)} \tilde{f}_{N}^{(N)}\left(\phi_{N}\right)=h_{m} h_{N}\left(L^{-2(N-m)} \cdot\right) \mathfrak{s}^{-(N-m)} \phi_{N}=h_{m} \mathfrak{s}^{-(N-m)} \phi_{N}
$$

since $f_{N}^{(N)}\left(\phi_{N}\right)=\phi_{N}$ by (31). Now $\phi_{N} \in B_{N}$ solves (191) with $\tilde{w}_{N}^{(N)}(\phi)=h_{N} w_{N}^{(N)}(\phi)$ with $w_{N}^{(N)}$ given by (30). Since $h_{N}=1$ on $\left[0, \tau_{N}-L^{-2}\right]$ we obtain

$$
\phi_{N}=\partial_{x} G_{1} *\left(\tilde{w}_{N}^{(N)}\left(\phi_{N}\right)+\xi_{N}\right)=\partial_{x} G_{1} *\left(w_{N}^{(N)}\left(\phi_{N}\right)+\Xi\right) .
$$

To take the limit $N \rightarrow \infty$ we will use (94): defining $\eta^{(N)}:=\mathfrak{s}^{-N} \phi_{N}$, then we get

$$
\eta^{(N)}=\mathfrak{s}^{-m} \tilde{f}_{m}^{(N)}(0)
$$

on the time interval $\left[0, \frac{1}{2} L^{-2 m}\right] \subset[0,1]$.

By Proposition $11 \tilde{f}_{m}^{(N)}(0)$ converges in $\mathcal{V}_{m}$ to a limit $\psi_{m}$ which is independent of the short distance cutoff. Convergence in $\mathcal{V}_{m}$ implies convergence in $\mathcal{D}^{\prime}\left([0,1] \times \mathbb{T}_{m}\right)$. The claim follows from continuity of $\mathfrak{s}^{-m}: \mathcal{D}^{\prime}\left([0,1] \times \mathbb{T}_{m}\right) \rightarrow \mathcal{D}^{\prime}\left(\left[0, L^{-2 m}\right] \times \mathbb{T}_{1}\right)$ and from the fact that convergence of $\eta=\partial_{x} u$ implies convergence of $u$.

\section{Proof of Proposition 7}

We now need to show that for some $\gamma>0$ the conditions defining the set $\mathcal{E}_{m}$ hold almost surely for some $m<\infty$. To do this, as in [5] the strategy is to control the covariances of the various fields in (61) and establish enough regularity for them.

We will deduce Proposition 7 from a covariance bound for the fields in (61). Let $\zeta_{n}^{(N)}(t, x)$ or $\zeta_{n}^{(N)}(t, x, s, y)$ be any of the fields in (61). Let

$$
\tilde{K}_{n}\left(t^{\prime}, t, x\right)=e^{\frac{1}{2} \operatorname{dist}\left(t^{\prime}, I_{n}\right)} K\left(t^{\prime}-t, x\right) h_{n}(t)
$$

where $I_{n}=\left[0, L^{2(n-m)}\right]$ and define

$$
\rho_{n}^{(N)}=\tilde{K}_{n} \zeta_{n}^{(N)} \quad \text { or } \quad \rho_{n}^{(N)}=\tilde{K}_{n} \otimes \tilde{K}_{n} \zeta_{n}^{(N)} .
$$

Then

$$
\left\|K \tilde{\zeta}_{n}^{(N)}\right\|_{L^{2}\left(\mathfrak{c}_{i}\right)} \leq C e^{-\frac{1}{2} \operatorname{dist}\left(i_{0}, I\right)}\left\|\rho_{n}^{(N)}\right\|_{L^{2}\left(\mathfrak{c}_{i}\right)} .
$$

where $i_{0}$ is the time component of the center of the cube $\mathfrak{c}_{i}$. From now on in the random fields we will drop the superscrip $(N)$ referring to the ultraviolet cutoff and we recall that $\|\cdot\|$ indicates the euclidean norm for a three-dimensional vector and the HilbertSchimdt norm for $3 \times 3$-matrix. The following proposition proved in Section 8.1 provides bounds for the covariance of $\rho_{n}$. 
Proposition 13. There exist renormalization constants $m_{1}, m_{2}, m_{3} \in \mathbb{R}^{3}$ and $\lambda>0$ such that for all $0 \leq n \leq N<\infty$ and for some constant $0<c<\frac{1}{2}$

$$
\begin{aligned}
\mathbb{E}\left\|\rho_{n}(t, x)\right\|^{2} & \leq C \\
\mathbb{E}\left\|\rho_{n}^{\prime}(t, x)-\rho_{n}(t, x)\right\|^{2} & \leq C L^{-\nu(N-n)}\left\|\chi-\chi^{\prime}\right\|_{\infty} \\
\mathbb{E} \| \rho_{n}\left(t, x, s, y \|^{2}\right. & \leq C e^{-c(|t-s|+|x-y|)} \\
\mathbb{E}\left\|\rho_{n}^{\prime}(t, x, s, y)-\rho_{n}(t, x, s, y)\right\|^{2} & \leq C L^{-\lambda(N-n)} e^{-c(|t-s|+|x-y|)}\left\|\chi-\chi^{\prime}\right\|_{\infty}
\end{aligned}
$$

where $\rho_{n}^{\prime}=\tilde{K} \zeta_{n}^{\prime}$, i.e. we replace the lower cutoff function $\chi$ by a $\chi^{\prime}$.

Now we can prove Proposition 7 ; we recall that we want to show that there exist $0 \leq m<\infty$ such that the event $\mathcal{E}_{m}$ holds almost surely, where $\mathcal{E}_{m}$ is the event such that bounds (65), (66) and (67) hold for any $m \leq n \leq N$. By using the same strategy as in [5] based on the bounds in [12, 13, one can see that Proposition 13 implies the following bounds for the random fields $\zeta_{n}^{(N)}$ in (61) for all $p>1$

$$
\begin{aligned}
& \mathbb{P}\left(\left\|\tilde{\zeta}_{n}^{(N)}\right\|_{\mathcal{V}_{n}} \geq L^{\gamma n}\right) \leq C L^{-2 m} L^{(3-2 \gamma p) n} \\
& \mathbb{P}\left(\left\|\tilde{\zeta}_{n}^{(N)}-\tilde{\zeta}_{n}^{(N)}\right\|_{\mathcal{V}_{n}} \geq L^{-\frac{1}{2} \gamma(N-n)} L^{\gamma n}\right) \leq C L^{-p \gamma(N-n)} L^{(3-2 \gamma p) n} L^{-2 m}
\end{aligned}
$$

Furthermore, to deal with the last condition on $\mathcal{E}_{m}$ in (67), we note that $\zeta:=\Upsilon_{n} * \xi_{n-1}$ is a Gaussian field with covariance

$$
\mathbb{E} \zeta\left(t^{\prime}, x^{\prime}\right) \zeta(t, x)=-\Delta_{x^{\prime}} \int_{0}^{\infty} H_{n}\left(t^{\prime}-t+2 s, x^{\prime}-x\right) \chi\left(t^{\prime}-t+s\right) \chi(s) d s
$$

where $\chi$ is smooth with support in $\left[L^{-2}, 2\right] . \mathbb{E} \zeta\left(t^{\prime}, x^{\prime}\right) \zeta(t, x)$ is smooth, compactly supported in $t^{\prime}-t$ and exponentially decaying in $x^{\prime}-x$. We get then by standard Gaussian estimates [12] for $0 \leq j \leq 2$ and $0 \leq j^{\prime} \leq 2$ and for some $c(L)>0$

$$
\mathbb{P}\left(\sup _{\alpha}\left\|\partial_{t}^{j} \partial_{x}^{j^{\prime}}\left(\Upsilon_{n} * \xi_{n-1}\right)_{\alpha}\right\|_{L^{\infty}\left(\mathfrak{c}_{i}\right)}>R\right) \leq C e^{-c(L) R^{2}}
$$

and thus

$$
\mathbb{P}\left(\sup _{\alpha}\left\|\left(\Upsilon_{n} * \xi_{n-1}\right)_{\alpha}\right\|_{\Phi_{n}}>L^{2 \gamma n}\right) \leq C L^{-2 m} L^{3 n} e^{-c(L) L^{4 \gamma n}} .
$$

The bounds (101), (102) and (104) implies that $\mathbb{P}\left(\mathcal{E}_{m}^{c}\right) \leq C L^{-2 m}$, then Proposition 7 follows from Borel-Cantelli Lemma.

8.1. Proof of Proposition 13. We will now study the random fields in (61), i.e.

$$
\zeta_{n} \in\left\{\vartheta_{n}, \mathfrak{z}_{n, i}, D \mathfrak{z}_{n, 2}\right\}
$$

that enter the probabilistic estimates.

Consider first their expectations. Setting $z=(t, x)$ and using Lemma 4 , the first one gives $\mathbb{E}_{\mathfrak{z}_{n, 1}}=\delta_{n} \leq C$, while for the second order fields we have

$$
\mathbb{E}_{\mathfrak{z} n, 1}=\delta_{n}, \quad \mathbb{E} \sigma_{\alpha \beta, n}\left(z, z^{\prime}\right)=\mathfrak{m}_{\alpha \beta} Y_{n}\left(z-z^{\prime}\right) \mathfrak{C}_{n}\left(z-z^{\prime}\right)
$$

and finally for the third order field we get

$$
\begin{aligned}
\mathbb{E}_{\mathfrak{z} n, 3}= & 8 \mathcal{M}_{1} \int d z_{1} d z_{2} Y_{n}\left(z_{2}\right) Y_{n}\left(z_{1}-z_{2}\right) \mathfrak{C}_{n}\left(z_{1}-z_{2}\right) \theta\left(t_{1}-t_{2}\right) \mathfrak{C}_{n}\left(z_{1}\right) \\
& +2 \mathcal{M}_{2} \int d z_{1} d z_{2} Y_{n}\left(z_{1}\right) Y_{n}\left(z_{2}\right) \mathfrak{C}_{n}\left(z_{1}-z_{2}\right)^{2}-m_{2} \log L^{N}-m_{3}
\end{aligned}
$$


where $\theta(t)=\mathbf{1}_{t \geq 0}$ is the Heaviside fuction and

$$
\begin{aligned}
\mathfrak{m}_{\alpha \beta} & =\sum_{\gamma, \delta} M_{\gamma \delta}^{(\alpha)} M_{\gamma \beta}^{(\delta)} \\
\left(\mathcal{M}_{1}\right)_{\alpha} & =\sum_{\beta_{1} \beta_{2} \beta_{3} \beta_{4}} M_{\beta_{1} \beta_{2}}^{(\alpha)} M_{\beta_{3} \beta_{4}}^{\left(\beta_{2}\right)} M_{\beta_{1} \beta_{3}}^{\left(\beta_{4}\right)} \\
\left(\mathcal{M}_{2}\right)_{\alpha} & =\sum_{\beta_{1} \beta_{2} \beta_{3} \beta_{4}} M_{\beta_{1} \beta_{2}}^{(\alpha)} M_{\beta_{3} \beta_{4}}^{\left(\beta_{2}\right)} M_{\beta_{3} \beta_{4}}^{\left(\beta_{1}\right)} .
\end{aligned}
$$

Define the random field

$$
\omega_{\alpha \beta}:=\vartheta_{\alpha} \vartheta_{\beta}-\mathbb{E} \vartheta_{\alpha} \vartheta_{\beta}
$$

(here and below $\vartheta=\vartheta_{n}^{(N)}$ ). Then the local fields $\zeta_{n}$ are linear combinations of their expectations and the following random fields

$$
\begin{gathered}
\vartheta_{\alpha}, \quad \omega_{\alpha \beta}, \quad Y_{n} * \omega_{\alpha \beta}, \quad Y_{n} * \omega_{\alpha \beta} Y_{n} * \omega_{\gamma \delta}-\mathbb{E} Y_{n} * \omega_{\alpha \beta} Y_{n} * \omega_{\gamma \delta}, \\
\vartheta_{\alpha} Y_{n} * \omega_{\beta \gamma}, \quad \vartheta_{\alpha} Y_{n} *\left(\vartheta_{\beta} Y_{n} * \omega_{\gamma \delta}\right)-\mathbb{E} \vartheta_{\alpha} Y_{n} *\left(\vartheta_{\beta} Y_{n} * \omega_{\gamma \delta}\right)
\end{gathered}
$$

where we used $Y_{n} * \delta_{n}=0$, while for the bi-local fields we need to consider

$$
Y_{n}\left(z-z^{\prime}\right) \vartheta_{\alpha}(z) \vartheta_{\beta}\left(z^{\prime}\right)-\mathbb{E} Y_{n}\left(z-z^{\prime}\right) \vartheta_{\alpha}(z) \vartheta_{\beta}\left(z^{\prime}\right)
$$

To get the covariance estimates for the fields (109), (110), (111) claimed in Proposition 13 we need to introduce the mixed covariance $\mathfrak{C}_{n}^{\prime}(z)$ such that

$$
\delta_{\alpha \beta} \mathfrak{C}_{n}^{\prime \prime}(z):=\mathbb{E} \vartheta_{\alpha}^{\prime}(z) \vartheta_{\beta}(0)
$$

where, as before, the primed kernels and fields have the lower cutoff $\chi^{\prime}$. Furthermore, let us define

$$
\begin{aligned}
\mathcal{C}_{n}(z) & :=\sup _{N \geq n}\left|\mathfrak{C}_{n}^{\prime}(z)\right| \\
\delta \mathcal{C}_{n}(z) & :=\left|\mathfrak{C}_{n}^{\prime}(z)-\mathfrak{C}_{n}(z)\right| \\
\mathcal{Y}_{n}(z) & :=\sup _{N \geq n}\left|Y_{n}(z)\right| \\
\delta \mathcal{Y}_{n}(z) & :=\left|Y_{n}^{\prime}(z)-Y_{n}(z)\right|
\end{aligned}
$$

The regularity of these kernels is summarized in the following Lemma proven in the Appendix.

Lemma 14. (a) For $p<3$ and uniformly in $n$ one has $\mathcal{C}_{n} \in L^{p}\left(\mathbb{R} \times \mathbb{T}_{n}\right)$ and

$$
\left\|\delta \mathcal{C}_{n}\right\|_{p}^{p} \leq C L^{-\lambda_{p}(N-n)}\left\|\chi-\chi^{\prime}\right\|_{\infty}
$$

for some $\lambda_{p}>0$.

(b) For $p<\frac{3}{2}$ and uniformly in $n$ one has $\mathcal{Y}_{n} \in L^{p}\left(\mathbb{R} \times \mathbb{T}_{n}\right)$ and

$$
\left\|\delta \mathcal{Y}_{n}\right\|_{p}^{p} \leq C L^{-\lambda_{p}(N-n)}\left\|\chi-\chi^{\prime}\right\|_{\infty}
$$

for some $\lambda_{p}>0$.

Having these technical tools at hand, we can finally start to show the covariance estimates. 
8.2. Fields (109) and (111). For $z=(t, x)$ we will use the norm $|z|=|t|+|x|$ and we will drop the subscript $n$ from the random fields and kernels. From the definition of the smoothing kernel $\tilde{K}$ we note that

$$
\tilde{K}\left(z, z^{\prime}\right) \leq C e^{-\frac{1}{2}\left|z-z^{\prime}\right|}, \quad \partial_{x} \tilde{K}\left(z, z^{\prime}\right) \leq C e^{-\frac{1}{2}\left|z-z^{\prime}\right|}, \quad \partial_{x^{\prime}} \tilde{K}\left(z, z^{\prime}\right) \leq C e^{-\frac{1}{2}\left|z-z^{\prime}\right|} .
$$

Defining

$$
X\left(z_{1}-z_{2}\right):=\mathbb{E} \zeta\left(z_{1}\right) \zeta\left(z_{2}\right)
$$

we then get

$$
\mathbb{E}\|\rho(z)\|^{2}=\int d z_{1} d z_{2} \tilde{K}\left(z, z_{1}\right) \tilde{K}\left(z, z_{2}\right) X\left(z_{1}-z_{2}\right) \leq C\|X\|_{1}
$$

i.e. it suffices to bound the $L^{1}$-norm of the covariance. We will use repeatedly the Young inequality in the form

$$
\left\|f_{1} * f_{2} * \cdots * f_{m}\right\|_{p} \leq \prod_{i=1}^{m}\left\|f_{i}\right\|_{p_{i}}
$$

if $n-1+\frac{1}{p}=\sum \frac{1}{p_{i}}$ where $1 \leq p, p_{i} \leq \infty$. We consider now the fields one by one.

(i) For $\zeta=\vartheta_{\alpha}$ we have $\|X\|_{1} \leq C\|\mathcal{C}\|_{1}$.

(ii) For $\zeta=\omega_{\alpha \beta}$ we have $\|X\|_{1} \leq C\|\mathcal{C}\|_{2}^{2}$.

(iii) For $\zeta=Y * \omega_{\alpha \beta}$ let $Y^{t}(z)=Y(-z)$. Then $X=C Y * \mathfrak{C} * Y^{t}$. By Young inequality

$$
\|X\|_{1} \leq C\left\|\mathcal{Y} * \mathcal{Y} * \mathcal{C}^{2}\right\|_{1} \leq C\|\mathcal{Y}\|_{1}^{2}\|\mathcal{C}\|_{2}^{2}
$$

(iv) For $\zeta=Y * \omega_{\alpha \beta} Y * \omega_{\gamma \delta}-\mathbb{E} Y * \omega_{\alpha \beta} Y * \omega_{\gamma \delta}$ we get

$$
\begin{aligned}
& \int d z \mathbb{E} \zeta(z) \zeta(0) \leq C \int d z d z_{1} \ldots d z_{4} \mathcal{Y}\left(z-z_{1}\right) \mathcal{Y}\left(z-z_{2}\right) \mathcal{Y}\left(-z_{3}\right) \mathcal{Y}\left(-z_{4}\right) \\
& \times\left[\mathcal{C}\left(z_{1}-z_{3}\right) \mathcal{C}\left(z_{2}-z_{4}\right)\left(\mathcal{C}\left(z_{1}-z_{4}\right) \mathcal{C}\left(z_{2}-z_{3}\right)+\mathcal{C}\left(z_{1}-z_{2}\right) \mathcal{C}\left(z_{3}-z_{4}\right)\right)\right. \\
& \left.+\mathcal{C}^{2}\left(z_{1}-z_{3}\right) \mathcal{C}^{2}\left(z_{2}-z_{4}\right)+\mathcal{C}^{2}\left(z_{1}-z_{4}\right) \mathcal{C}^{2}\left(z_{2}-z_{3}\right)\right]
\end{aligned}
$$

Using the trivial inequality

$$
2|a b| \leq a^{2}+b^{2}
$$

with $a, b \in \mathbb{R}$ for the products of $\mathcal{C}$, we obtain

$$
\begin{aligned}
\int d z \mathbb{E} \zeta(z) \zeta(0) \leq C & \int d z d z_{1} \ldots d z_{4} \mathcal{Y}\left(z-z_{1}\right) \mathcal{Y}\left(z-z_{2}\right) \mathcal{Y}\left(-z_{3}\right) \mathcal{Y}\left(-z_{4}\right) \\
& \times\left[\mathcal{C}\left(z_{1}-z_{3}\right) \mathcal{C}\left(z_{2}-z_{4}\right)\left(\mathcal{C}\left(z_{1}-z_{4}\right)^{2}+\mathcal{C}\left(z_{2}-z_{3}\right)^{2}\right)\right. \\
& +\mathcal{C}\left(z_{1}-z_{2}\right) \mathcal{C}\left(z_{3}-z_{4}\right)\left(\mathcal{C}\left(z_{1}-z_{3}\right)^{2}+\mathcal{C}\left(z_{2}-z_{4}\right)^{2}\right) \\
& \left.+\mathcal{C}^{2}\left(z_{1}-z_{3}\right) \mathcal{C}^{2}\left(z_{2}-z_{4}\right)+\mathcal{C}^{2}\left(z_{1}-z_{4}\right) \mathcal{C}^{2}\left(z_{2}-z_{3}\right)\right]
\end{aligned}
$$

Note that $\mathcal{C}_{n} \in L^{p}$ with $p<\frac{3}{2}$ thanks to Lemma 14, so by Young inequality one can see that the first two terms in (120) are bounded by

$$
\begin{aligned}
& C \|(\mathcal{Y} * \mathcal{C})\left(\mathcal{Y} *\left(\mathcal{C}_{n}^{2}(\mathcal{Y} * \mathcal{Y} * \mathcal{C})\right)\left\|_{1} \leq C\right\| \mathcal{Y} * \mathcal{C}\left\|_{2}\right\| \mathcal{Y} * \mathcal{C}^{2}(\mathcal{Y} * \mathcal{Y} * \mathcal{C}) \|_{2}\right. \\
& \leq C\|\mathcal{Y}\|_{1}\|\mathcal{C}\|_{2}\|\mathcal{Y}\|_{\frac{4}{3}}\left\|\mathcal{C}^{2}\right\|_{\frac{4}{3}}\|\mathcal{Y} * \mathcal{Y} * \mathcal{C}\|_{\infty} \leq C\|\mathcal{Y}\|_{1}\|\mathcal{Y}\|_{\frac{4}{3}}^{3}\|\mathcal{C}\|_{2}^{2}\left\|\mathcal{C}^{2}\right\|_{\frac{4}{3}}
\end{aligned}
$$


while the third and fourth term in (120) are bounded by

$$
\begin{aligned}
& C\|\mathcal{C}(\mathcal{Y} * \mathcal{Y})\|_{1}\left\|\mathcal{C}^{2} *(\mathcal{Y}(\mathcal{Y} * \mathcal{C}))\right\|_{1} \leq C\|\mathcal{C}\|_{2}\|\mathcal{Y} * \mathcal{Y}\|_{2}\left\|\mathcal{C}^{2}\right\|_{1}\|\mathcal{Y}(\mathcal{Y} * \mathcal{C})\|_{1} \\
& \leq C\|\mathcal{C}\|_{2}^{4}\|\mathcal{Y}\|_{\frac{4}{3}}^{4}
\end{aligned}
$$

and the last constributions are bounded by

$$
C\left\|\mathcal{Y} * \mathcal{Y} * \mathcal{Y} * \mathcal{C}^{2}\right\|_{2}\left\|\mathcal{C}^{2} * \mathcal{Y}\right\|_{2} \leq C\|\mathcal{Y}\|_{1}^{2}\|\mathcal{Y}\|_{\frac{4}{3}}^{2}\left\|\mathcal{C}^{2}\right\|_{\frac{4}{3}}^{2}
$$

(v) Next we consider the bi-local field $\zeta\left(z_{1}, z_{2}\right)=Y\left(z_{1}-z_{2}\right)\left(\vartheta_{\alpha}\left(z_{1}\right) \vartheta_{\beta}\left(z_{2}\right)-\delta_{\alpha \beta} \mathfrak{C}\left(z_{1}-z_{2}\right)\right)$. Then we have

$\mathbb{E} \zeta\left(z_{1}, z_{2}\right) \zeta\left(z_{3}, z_{4}\right) \leq C \mathcal{Y}\left(z_{1}-z_{2}\right) \mathcal{Y}\left(z_{3}-z_{4}\right)\left(\mathcal{C}\left(z_{1}-z_{3}\right) \mathcal{C}\left(z_{2}-z_{4}\right)+\mathcal{C}\left(z_{1}-z_{4}\right) \mathcal{C}\left(z_{2}-z_{3}\right)\right)$

so that

$$
\begin{aligned}
& e^{c\left|z_{1}-z_{2}\right|} \mathbb{E}\left\|\rho_{n}\left(z_{1}, z_{2}\right)\right\|^{2} \leq C e^{c\left|z_{1}-z_{2}\right|} \mid \int d z_{1234}^{\prime} \tilde{K}\left(z_{1}, z_{1}^{\prime}\right) \tilde{K}\left(z_{2}, z_{2}^{\prime}\right) \tilde{K}\left(z_{1}, z_{3}^{\prime}\right) \tilde{K}\left(z_{2}, z_{4}^{\prime}\right) \\
& \times \mathcal{Y}\left(z_{1}^{\prime}-z_{2}^{\prime}\right) \mathcal{Y}\left(z_{3}^{\prime}-z_{4}^{\prime}\right)\left[\mathcal{C}\left(z_{1}^{\prime}-z_{3}^{\prime}\right) \mathcal{C}_{n}\left(z_{2}^{\prime}-z_{4}^{\prime}\right)+\mathcal{C}_{n}\left(z_{1}^{\prime}-z_{4}^{\prime}\right) \mathcal{C}_{n}\left(z_{2}^{\prime}-z_{3}^{\prime}\right)\right] \mid
\end{aligned}
$$

where $0<c<\frac{1}{2}$ and then

$$
\mathbb{E}\left\|\rho_{n}\left(z_{1}, z_{2}\right)\right\|^{2} \leq C e^{-c\left|z_{1}-z_{2}\right|}\|\tilde{\mathcal{Y}} * \mathcal{Y} * \mathcal{C} * \mathcal{C}\|_{1} \leq C e^{-c\left|z_{1}-z_{2}\right|}\|\tilde{\mathcal{Y}}\|_{1}\|\mathcal{Y}\|_{1}\|\mathcal{C}\|_{1}^{2}
$$

where $\tilde{\mathcal{Y}}(z):=e^{c|z|} \mathcal{Y}(z)$ is in $L^{p}$ with $p<\frac{3}{2}$.

8.3. Fields (110) and (105). We observe that in the above covariance estimates, the Young inequality trick requires all the kernels to be at least in $L^{1}\left(\mathbb{R} \times \mathbb{T}_{n}\right)$. Unfortunately in the fields (110) and (105) the kernel $J_{n}(z):=Y_{n}(z) \mathfrak{C}_{n}(z)$ will appear and it is easy to see that $\left\|J_{n}\right\|_{1}$ diverges logarithmically as $N \rightarrow 0$, so Young inequality cannot be applied as before.

The following Lemma shows some properties of $J_{n}$ which are crucial to overcome this problem. Its proof can be found in the Appendix.

Lemma 15. (a) We have

$$
J_{n}(z)=\partial_{x} W_{n}(z)+j_{n}(z)
$$

where $W_{n}$ is in $L^{1}\left(\mathbb{R} \times \mathbb{T}_{n}\right)$ uniformly in $n, N$ and

$$
\left|j_{n}(z)\right| \leq C e^{-|x|} \mathbf{1}_{[0,2]}(t) .
$$

(b) The function $Z_{n}:=Y_{n} * J_{n}$ is in $L^{1}\left(\mathbb{R} \times \mathbb{T}_{n}\right)$ uniformly in $n, N$.

(c) $\left\|W_{n}-W_{n}^{\prime}\right\|_{1} \leq C L^{-\lambda(N-n)}$ for some $\lambda>0$, idem for $j_{n}$ and $Z_{n}$.

(d) Let be $\epsilon=L^{-2(N-n)}$, then

$$
\begin{gathered}
\left|W_{n}(z)-\mathfrak{C}_{n}(z)^{2}\right| \leq C\left(\epsilon^{-2} e^{-c|x| / \epsilon} \mathbf{1}_{\left[\frac{1}{2} \epsilon^{2}, 2 \epsilon^{2}\right]}(t)+e^{-c|x|} \mathbf{1}_{\left[\frac{1}{2}, 2\right]}(t)\right) \\
\left|Y_{n}(z)-2 \partial_{x} \mathfrak{C}_{n}(z)\right| \leq C\left(\epsilon^{-2} e^{-c|x| / \epsilon} \mathbf{1}_{\left[\frac{1}{2} \epsilon^{2}, 2 \epsilon^{2}\right]}(t)+e^{-c|x|} \mathbf{1}_{\left[\frac{1}{2}, 2\right]}(t)\right) .
\end{gathered}
$$

In practice Lemma 15 guarantees that the nasty kernel $J_{n}$ is actually a gradient of an $L^{1}$-function, up to to a smooth correction. By an integration by parts this property will allow us to move the gradient and make it act on the smoothing kernels $\tilde{K}$, so that we can still use the Young inequality. Moreover, we point out that the kernel $Z_{n}$ will 
appear in the last fields in (110). Finally, item (d) in Lemma 15 will be employed to study the divergence of $\mathbb{E}_{\mathfrak{z} n, 3}$ in (106).

In the following we will neglect the remainder term $j(z)$, since its contributions can be easily bounded as we have done for the fields in (109).

(vi) For $\zeta=\vartheta_{\alpha} Y * \omega_{\beta \gamma}$ we have

$$
\begin{aligned}
\mathbb{E}\|\rho(z)\|^{2} \leq & C\left|\int d z_{1234} \tilde{K}\left(z, z_{1}\right) \tilde{K}\left(z, z_{2}\right) J\left(z_{1}-z_{3}\right) J\left(z_{2}-z_{4}\right) \mathfrak{C}\left(z_{3}-z_{4}\right)\right| \\
& +C \int d z d z_{1} d z_{2} \mathcal{Y}\left(z-z_{1}\right) \mathcal{Y}\left(-z_{2}\right)\left[\mathcal{C}(z) \mathcal{C}^{2}\left(z_{1}-z_{2}\right)+\mathcal{C}\left(z-z_{2}\right) \mathcal{C}\left(z_{1}\right) \mathcal{C}\left(z_{1}-z_{2}\right)\right]
\end{aligned}
$$

Using Lemma 15, (115) and Young inequality we get

$$
\begin{aligned}
\mathbb{E}\|\rho(z)\|^{2} & \leq C\left[\|W\|_{1}\|\mathcal{C} * W\|_{1}+\|\mathcal{C} * \mathcal{Y}\|_{2}\left\|\mathcal{C}^{2} * \mathcal{Y}\right\|_{2}+\|\mathcal{C}(\mathcal{C} * \mathcal{Y}) * \mathcal{Y}\|_{2}\|\mathcal{C}\|_{2}\right] \\
& \leq C\left[\|W\|_{1}^{2}\|\mathcal{C}\|_{1}+\|\mathcal{Y}\|_{1}\|\mathcal{C}\|_{2}\left\|\mathcal{C}^{2}\right\|_{\frac{4}{3}}\|\mathcal{Y}\|_{\frac{4}{3}}+\|\mathcal{Y}\|_{1}\|\mathcal{Y}\|_{\frac{4}{3}}\|\mathcal{C}\|_{2}\left\|\mathcal{C}^{2}\right\|_{\frac{4}{3}}\right]
\end{aligned}
$$

For the last field in (110), i.e. $\vartheta_{\alpha} Y *\left(\vartheta_{\beta} Y * \omega_{\gamma \delta}\right)-\mathbb{E} \vartheta_{\alpha} Y *\left(\vartheta_{\beta} Y * \omega_{\gamma \delta}\right)$ it is convenient to perform an expansion in terms of Wick polynomials to keep track of the several contributions involved in the covariance (see [14] for a recent review about Wick polynomials). In our case the "elementary" fields $\vartheta_{n}$ are Gaussian and with vanishing expectation value, so the combinatorics of the Wick expansion will be quite simple. Noting that $\omega_{\alpha \beta}=: \vartheta_{\alpha} \vartheta_{\beta}$ :, the random fields turns out to be a linear combination of the following terms

$$
\begin{aligned}
& : \vartheta_{\alpha} Y *\left(\vartheta_{\beta} Y * \vartheta_{\gamma} \vartheta_{\delta}\right):, \quad Z * \omega_{\alpha \beta}, \quad: \vartheta_{\alpha} Z * \vartheta_{\beta}: \\
& \int d z_{1} d z_{2} Y\left(z-z_{1}\right) Y\left(z_{1}-z_{2}\right) \mathfrak{C}\left(z-z_{2}\right): \vartheta_{\alpha}\left(z_{1}\right) \vartheta_{\beta}\left(z_{2}\right):
\end{aligned}
$$

(vii) In $\zeta=: \vartheta_{\alpha} Y *\left(\vartheta_{\beta} Y * \vartheta_{\gamma} \vartheta_{\delta}\right)$ : there is no $J$ appearing, so we can just estimate the corresponding $\|X\|_{1}$ which unfortunately has many terms:

$$
\begin{aligned}
& \|X\|_{1} \leq C \int d z d z_{1} \cdots d z_{4} \mathcal{Y}\left(z-z_{1}\right) \mathcal{Y}\left(z_{1}-z_{2}\right) \mathcal{Y}\left(-z_{3}\right) \mathcal{Y}\left(z_{3}-z_{4}\right) \\
& \times\left[\mathcal{C}(z) \mathcal{C}\left(z_{1}-z_{3}\right) \mathcal{C}^{2}\left(z_{2}-z_{4}\right)+\mathcal{C}\left(z-z_{3}\right) \mathcal{C}\left(z_{1}\right) \mathcal{C}^{2}\left(z_{2}-z_{4}\right)\right. \\
& +\mathcal{C}(z) \mathcal{C}\left(z_{1}-z_{4}\right) \mathcal{C}\left(z_{2}-z_{3}\right) \mathcal{C}\left(z_{2}-z_{4}\right)+\mathcal{C}\left(z-z_{3}\right) \mathcal{C}\left(z_{1}-z_{4}\right) \mathcal{C}\left(z_{2}\right) \mathcal{C}\left(z_{2}-z_{4}\right) \\
& +\mathcal{C}\left(z-z_{4}\right) \mathcal{C}\left(z_{1}\right) \mathcal{C}\left(z_{2}-z_{3}\right) \mathcal{C}\left(z_{2}-z_{4}\right)+\mathcal{C}\left(z-z_{4}\right) \mathcal{C}\left(z_{1}-z_{3}\right) \mathcal{C}\left(z_{2}\right) \mathcal{C}\left(z_{2}-z_{4}\right) \\
& \left.+\mathcal{C}\left(z-z_{4}\right) \mathcal{C}\left(z_{1}-z_{4}\right) \mathcal{C}\left(z_{2}-z_{3}\right) \mathcal{C}\left(z_{2}\right)\right]
\end{aligned}
$$

Using (119) we get

$$
\begin{aligned}
& \|X\|_{1} \leq C\left[\left\|\mathcal{C}\left(\mathcal{Y} * \mathcal{Y} * \mathcal{C}^{2}\right)\right\|_{\frac{4}{3}}\|\mathcal{C} * \mathcal{Y}\|_{4}+\|\mathcal{C}\|_{2}\left\|\mathcal{Y} *(\mathcal{Y} * \mathcal{C})\left(\mathcal{Y} * \mathcal{Y} * \mathcal{C}^{2}\right)\right\|_{2}\right. \\
& +\|\mathcal{C} * \mathcal{Y}\|_{4}\left\|\mathcal{Y} *(\mathcal{Y} * \mathcal{C})\left(\mathcal{Y} * \mathcal{C}^{2}\right)\right\|_{\frac{4}{3}}+\|\mathcal{Y} * \mathcal{C}\|_{2}\left\|\mathcal{Y} * \mathcal{Y} * \mathcal{C}\left(\mathcal{Y} * \mathcal{C}^{2}\right)\right\|_{2} \\
& +\left\|\mathcal{Y} * \mathcal{C}^{2}\right\|_{2}\left\|\mathcal{Y} *(\mathcal{C} * \mathcal{Y})^{2}\right\|_{2}+\|\mathcal{Y}\|_{1}\left\|(\mathcal{C} * \mathcal{Y})\left(\mathcal{Y} * \mathcal{C}\left(\mathcal{C}^{2} * \mathcal{Y}\right)\right)\right\|_{1} \\
& +\left\|\mathcal{Y} * \mathcal{C}^{2}\right\|_{2}\|\mathcal{Y} * \mathcal{C}(\mathcal{Y} * \mathcal{Y} * \mathcal{C})\|_{2}+\|\mathcal{Y}\|_{1}\left\|\mathcal{C}\left(\mathcal{Y} *(\mathcal{C} * \mathcal{Y})\left(\mathcal{C}^{2} * \mathcal{Y}\right)\right)\right\|_{1} \\
& +\|\mathcal{Y}\|_{1}\left\|\left(\mathcal{Y} * \mathcal{C}^{2}\right)(\mathcal{Y} * \mathcal{C}(\mathcal{C} * \mathcal{Y}))\right\|_{1}+\left\|\mathcal{C}^{2} \mathcal{Y}\right\|_{2}\|\mathcal{Y} * \mathcal{Y} * \mathcal{C}(\mathcal{C} * \mathcal{Y})\|_{2} \\
& \leq C\left[\|\mathcal{Y}\|_{\frac{4}{3}}^{4}\|\mathcal{C}\|_{2}^{4}+\|\mathcal{Y}\|_{1}\|\mathcal{Y}\|_{\frac{4}{3}}^{3}\|\mathcal{C}\|_{2}^{2}\left\|\mathcal{C}^{2}\right\|_{\frac{4}{3}}\right]
\end{aligned}
$$


(viii) For $\zeta=Z * \omega_{\alpha \beta}$ we have by Lemma 15)(b)

$$
\|X\| \leq C\left\|Z * \mathcal{C}^{2}\right\|_{1}\|Z\|_{1} \leq C\|Z\|_{1}^{2}\left\|\mathcal{C}^{2}\right\|_{1} .
$$

(ix) For $\zeta=: \vartheta_{\alpha} Z * \vartheta_{\beta}$ : again by Lemma 15(b) we have

$$
\|X\| \leq C\|Z * \mathcal{C}\|_{2}^{2} \leq C\|Z\|_{1}^{2}\|\mathcal{C}\|_{2}^{2} .
$$

(x) For $\zeta=\int d z_{1} d z_{2} Y\left(z-z_{1}\right) Y\left(z_{1}-z_{2}\right) \mathfrak{C}\left(z-z_{2}\right): \vartheta_{\alpha}\left(z_{1}\right) \vartheta_{\beta}\left(z_{2}\right)$ : by (119) we have

$$
\begin{aligned}
\|X\|_{1} \leq & C \int d z d z_{1} \cdots d z_{4} \mathcal{Y}\left(z-z_{1}\right) \mathcal{Y}\left(z_{1}-z_{2}\right) \mathcal{C}\left(z-z_{2}\right) \mathcal{Y}\left(-z_{3}\right) \mathcal{Y}\left(z_{3}-z_{4}\right) \mathcal{C}\left(-z_{4}\right) \\
& \times\left[\mathcal{C}^{2}\left(z_{1}-z_{3}\right)+\mathcal{C}^{2}\left(z_{2}-z_{4}\right)+\mathcal{C}^{2}\left(z_{1}-z_{4}\right)+\mathcal{C}^{2}\left(z_{2}-z_{3}\right)\right] \\
\leq & C\|\mathcal{Y}\|_{\frac{4}{3}}^{4}\|\mathcal{C}\|_{2}^{4} .
\end{aligned}
$$

(xi) We still need to bound $\zeta\left(z_{1}-z_{2}\right)=\mathbb{E} \sigma_{\alpha \beta}\left(z_{1}, z_{2}\right)=\mathfrak{m}_{\alpha \beta} J\left(z_{1}-z_{2}\right)$. Using Lemma 15)(a) and the same strategy used for (v) we get

$$
e^{c\left|z_{1}-z_{2}\right|}\left\|\rho\left(z_{1}, z_{2}\right)\right\|^{2} \leq C\|\tilde{W}\|_{1}^{2}
$$

where $\tilde{W}(z)=e^{c|z|} W(z) \in L^{1}\left(\mathbb{R} \times \mathbb{T}_{n}\right)$.

We observe that the estimates (98) and (100) are obtained as the bounds (97) and (99) derived above by using Lemma 4, (113), (114) and Lemma 15)(c).

8.4. Third order renormalization. So we are left with the analysis of the expectation $\mathbb{E}_{\mathfrak{z}_{3}}$ which will allow us to determine the renormalization constants $m_{2}, m_{3}$ :

$$
\begin{aligned}
\mathbb{E}_{\mathfrak{z}_{3}} & =2 \mathcal{M}_{2} \int\left(Y * \mathfrak{C}^{2}\right)(z) Y(z) d z+8 \mathcal{M}_{1} \int(Y * \theta J)(z) \mathfrak{C}(z) d z-m_{2} \log L^{N}-m_{3} \\
& =4 \mathcal{M}_{2} \int\left(Y * \theta \mathfrak{C}^{2}\right)(z) Y(z) d z+8 \mathcal{M}_{1} \int(Y * \theta J)(z) \mathfrak{C}(z) d z-m_{2} \log L^{N}-m_{3}
\end{aligned}
$$

where $\left(\theta \mathfrak{C}^{2}\right)(z)=\theta(t) \mathfrak{C}^{2}(z)$ and similarly for $\theta J$. Let us call $\mathcal{A}=\partial_{x} \mathfrak{C}-\frac{1}{2} Y$ and $\mathcal{B}=W-\mathfrak{C}^{2}$. Using Lemma 15 and an integration by parts in the second term we get

$$
\begin{aligned}
\mathbb{E} \mathfrak{z}_{n, 3}= & 8 \mathcal{M}_{1} \int\left[(Y * \theta j)(z) \mathfrak{C}(z)-\left(Y * \theta \mathfrak{C}^{2}\right)(z) \mathcal{A}(z)-(Y * \theta \mathcal{B})(z) \mathfrak{C}(z)\right] d z-m_{3} \\
& +4\left(\mathcal{M}_{2}-\mathcal{M}_{1}\right) \int\left(Y * \theta \mathfrak{C}^{2}\right)(z) Y(z) d z-m_{2} \log L^{N}
\end{aligned}
$$

For the first term we use the bounds in Lemma 15 to get

$$
\left|\int\left[(Y * \theta j)(z) \mathfrak{C}(z)-\left(Y * \theta \mathfrak{C}^{2}\right)(z) \mathcal{A}(z)-(Y * \theta \mathcal{B})(z) \mathfrak{C}(z)\right] d z\right| \leq C .
$$


Let us now study the second term in (129) which is the divergent one. In Fourier space we have

$$
\begin{aligned}
& \int\left(Y * \theta \mathfrak{C}^{2}\right)(z) Y(z) d z \\
= & \int_{0}^{\infty} d t \int d p \widehat{Y}(t,-p) \int_{0}^{t} d s \widehat{Y}(t-s, p) \int d q \widehat{\mathfrak{C}}(s, p+q) \widehat{\mathfrak{C}}(s, q) \\
= & \int_{0}^{\infty} d t \chi_{\epsilon}(t) \int d p p^{2} e^{-t p^{2}} \int_{0}^{t} d s \chi_{\epsilon}(t-s) e^{-(t-s) p^{2}} \int d q e^{-s\left(q^{2}+(p+q)^{2}\right)} \\
& \times h_{\epsilon}(s, \sqrt{s}(p+q)) h_{\epsilon}(s, \sqrt{s} q)
\end{aligned}
$$

where

$$
h_{\epsilon}(t, p)=p^{2} \int_{0}^{\infty} d \sigma e^{-2 \sigma p^{2}} \chi_{\epsilon}((1+\sigma) t) \chi_{\epsilon}(\sigma t)
$$

and $0 \leq h_{\epsilon}<\frac{1}{2}$ uniformly on $\mathbb{R}_{+} \times \mathbb{R}$. Let us define $\mu_{\epsilon}$ as

$$
\mu_{\epsilon}:=\frac{1}{4} \int_{\epsilon^{2}}^{2} d t \int d p p^{2} e^{-t p^{2}} \int_{0}^{t} d s e^{-(t-s) p^{2}} \mathbf{1}_{\left[\epsilon^{2}, 2\right]}(t-s) \int d q e^{-s\left(q^{2}+(p+q)^{2}\right)} .
$$

We get that

$$
\begin{aligned}
0 \leq & \mu_{\epsilon}-\int\left(Y * \theta \mathfrak{C}^{2}\right)(z) Y(z) d z \\
\leq & \frac{1}{4} \int_{\epsilon^{2}}^{2} d t \int d p p^{2} e^{-t p^{2}} \int_{0}^{t} d s e^{-(t-s) p^{2}} \int d q e^{-s\left(q^{2}+(p+q)^{2}\right)} \\
& \times\left[\mathbf{1}_{\left[\epsilon^{2}, 2\right]}(t)\left(\mathbf{1}_{\left[\epsilon^{2}, 2 \epsilon^{2}\right]}(t-s)+\mathbf{1}_{[1,2]}(t-s)\right)+\mathbf{1}_{\left[\epsilon^{2}, 2\right]}(t-s)\left(\mathbf{1}_{\left[\epsilon^{2}, 2 \epsilon^{2}\right]}(t)+\mathbf{1}_{[1,2]}(t)\right)\right] \leq C \\
&
\end{aligned}
$$

Let us also define $\tilde{\mu}_{\epsilon}:=\frac{\pi}{4 \sqrt{3}} \log \epsilon^{-1}$, then by an explicit computation one gets

$$
\lim _{\epsilon \rightarrow 0}\left(\tilde{\mu}_{\epsilon}-\mu_{\epsilon}\right)=\mathcal{O}(1) .
$$

Therefore, we can identify the universal renormalization constant $m_{2}$ as

$$
m_{2}=4\left(\mathcal{M}_{2}-\mathcal{M}_{1}\right) \frac{\tilde{\mu}_{\epsilon}}{\log \epsilon^{-1}}=\frac{\pi}{\sqrt{3}}\left(\mathcal{M}_{2}-\mathcal{M}_{1}\right) .
$$

Finally, for the $\chi$-dependent renormalization constant $m_{3}$, let be $\nu_{\epsilon}:=\mathbb{E} \mathfrak{z}_{n, 3}-m_{2} \log \epsilon^{-1}$ : from (126), (134) and (135) we know that $\left|\nu_{\epsilon}\right| \leq C$ and by bounds similar to ones in Lemma 15 comparing different cutoffs one can see that $\nu_{\epsilon}$ is a Cauchy sequence. Therefore, in the end we obtain

$$
m_{3}=\lim _{\epsilon \rightarrow 0}\left(\mathbb{E}_{\mathfrak{z} n, 3}-m_{2} \log \epsilon^{-1}\right)=\mathcal{O}(1) .
$$

Remark 16 (Cancellation of the third order divergence). We observe that for some special class of vectors of symmetric matrices $M=\left(M^{(1)}, M^{(2)}, M^{(3)}\right)$ the normalization constants $m_{2}$ and $m_{3}$ are not needed, i.e. $m_{2}=m_{3}=0$ (for example this is the case of the ordinary $K P Z$ equation where $u \in \mathbb{R})$.

In fact, if $M_{\beta \gamma}^{(\alpha)}$ is totally symmetric with respect to three indices, i.e. it is also invariant under the swap $\alpha \leftrightarrow \beta$, then $\mathcal{M}_{1}=\mathcal{M}_{2}$ in (106) and (107) and the divergent term is not present. 
From (37) one has

$$
\mathbb{E}\left(\vartheta_{n}^{(N)}(t, x), M^{(\alpha)} \vartheta_{n}^{(N)}(t, x)\right)=\left(\sum_{\beta=1}^{3} M_{\beta \beta}^{(\alpha)}\right) \mathfrak{C}_{n}^{(N)}(0,0)
$$

Let us split $\mathfrak{C}_{n}^{(N)}(0,0)$ by isolating the term corresponding to $i=0$ in (35):

$$
\mathfrak{C}_{n}^{(N)}(0,0)=\frac{1}{2^{7 / 2} \sqrt{\pi}} \int_{0}^{\infty} \frac{\chi(s)^{2}-\chi^{\prime}\left(L^{2(N-n)} s\right)^{2}}{s^{3 / 2}} d s+R
$$

where to stress the cutoff dependence we wrote this with the lower cutoff $\chi^{\prime}$.

The remainder is easily bounded by

$$
R \leq C e^{-c L^{2 n}} .
$$

and its change with cutoff by

$$
\left|R-R^{\prime}\right| \leq C e^{-c L^{2 N}}\left\|\chi-\chi^{\prime}\right\|_{\infty} .
$$

For the main term in (138) we define

$$
\rho_{\chi}=\int_{0}^{\infty} \frac{1-\chi(s)^{2}}{s^{3 / 2}} d s .
$$

Then

$$
\int_{0}^{\infty} \frac{\chi(s)^{2}-\chi^{\prime}\left(L^{2(N-n)} s\right)^{2}}{s^{3 / 2}} d s=L^{N-n} \rho_{\chi^{\prime}}-\rho_{\chi}
$$

Setting $\delta_{n}^{(N)}=\sum_{\beta=1}^{3} M_{\beta \beta}^{(\alpha)}\left(R-\rho_{\chi}\right)$ the claim follows.

\section{Appendix B. Proof of Lemma 14}

(a) We have:

$$
\mathfrak{C}_{n}^{\prime}(t, x)=-\Delta \int_{0}^{\infty} H_{n}(t+2 s, x) \chi_{N-n}(t+s) \chi_{N-n}^{\prime}(s) d s
$$

where $\chi_{N-n}^{\prime}(t)=\chi(t)-\chi\left(L^{2(N-n)} t\right)$. Therefore, since $\chi_{N-n}(t+s) \chi_{N-n}^{\prime}(s) \leq \mathbf{1}_{[0,2]}(s) \mathbf{1}_{[0,2]}(t)$, one has

$$
\left|\mathfrak{C}_{n}^{\prime}(t, x)\right| \leq C \mathbf{1}_{[0,2]}(t) \sum_{j \in \mathbb{Z}} \ell\left(t, x+j L^{n}\right)
$$

where

$$
\begin{aligned}
\ell\left(t, x+j L^{n}\right) & \leq C \int_{0}^{2} d s(t+2 s)^{-\frac{3}{2}} e^{-\frac{x^{2}}{4(t+2 s)}}\left[1+x^{2}(t+2 s)^{-1}\right] \\
& \leq C e^{-c x^{2}}\left(x^{2}+t\right)^{-\frac{1}{2}}\left[1+x^{2}\left(x^{2}+t\right)^{-1}\right] \mathbf{1}_{[0,2]}(t)+e^{-c x^{2} / t} t^{-\frac{3}{2}}\left[1+x^{2} t^{-1}\right] \mathbf{1}_{[2, \infty)}(t)
\end{aligned}
$$

Combining (140) with (141) one gets

$$
\mathcal{C}_{n}(\tau, x) \leq C e^{-c x^{2}}\left(x^{2}+t\right)^{-\frac{1}{2}}\left[1+x^{2}\left(x^{2}+t\right)^{-1}\right] \mathbf{1}_{[0,2]}(t) \in L^{p}\left(\mathbb{R} \times \mathbb{T}_{n}\right)
$$

for $p<3$. To show (114), note that

$$
\chi_{N-n}(t+s)\left|\chi_{\epsilon}(s)-\chi_{N-n}^{\prime}(s)\right| \leq \mathbf{1}_{\left[\epsilon^{2}, 2 \epsilon^{2}\right]}(s) \mathbf{1}_{[0,2]}(t)\left\|\chi-\chi^{\prime}\right\|_{\infty}
$$


where $\epsilon=L^{-(N-n)}$. Hence

$$
\delta \mathcal{C}_{n}(t, x) \leq C \sum_{j \in \mathbb{Z}} \ell_{N-n}\left(t, x+j L^{n}\right) \mathbf{1}_{[0,2]}(t)\left\|\chi-\chi^{\prime}\right\|_{\infty}
$$

where

$$
\ell_{M}(t, x):=\int_{0}^{2 L^{-2 M}}(t+2 s)^{-\frac{3}{2}} e^{-\frac{x^{2}}{4(t+2 s)}}\left[1+x^{2}(t+2 s)^{-1}\right] d s=L^{M} \ell_{0}\left(L^{2 M} t, L^{M} x\right) .
$$

Hence using (141) we have

$$
\begin{aligned}
\left\|\ell_{M}(t, x) \mathbf{1}_{[0,2]}(t)\right\|_{p}^{p} & =L^{-(3-p) M}\left\|\ell_{0}(t, x) \mathbf{1}_{\left[0,2 L^{2 M}\right]}(t)\right\|_{p}^{p} \\
& \leq C L^{-(3-p) M}\left(1+\int_{2}^{2 L^{2 M}} t^{\frac{3}{2}(1-p)} d t\right) \leq C L^{-\lambda M}
\end{aligned}
$$

with $\lambda>0$ for $p<3$.

(b) The claim follows with the same strategy employed in item (a).

\section{Appendix C. Proof of Lemma 15}

First of all, we note that we can replace $H_{n}$ (the heat kernel on $\mathbb{T}_{n}$ ) by $H$ (the heat kernel on $\mathbb{R}$ ) in $\mathfrak{C}_{n}$ and $\mathcal{Y}_{n}$. Indeed, letting $\tilde{\mathcal{K}}$ denote the kernels $\mathfrak{C}_{n}, \mathcal{Y}_{n}$ and $J_{n}$ built out of $H$ we get

$$
|\tilde{\mathcal{K}}(z)-\mathcal{K}(z)| \leq C e^{-|x|} \mathbf{1}_{[0,2]}(t) .
$$

Therefore, in the following proof we will consider kernels built with $H$ and drop the tildes.

Let $\epsilon=L^{-(N-n)}$ and $\chi_{\epsilon}=\chi_{N-n}$. We will indicate the scale dependence of the kernels by $\epsilon$ instead of $n$, i.e. $\mathfrak{C}_{n}=\mathfrak{C}_{\epsilon}$ and so on. We work in Fourier space in the $x$ variable:

$$
\widehat{\mathfrak{C}}_{\epsilon}(t, p)=p^{2} e^{-t p^{2}} \int_{0}^{\infty} d s e^{-2 s p^{2}} \chi_{\epsilon}(t+s) \chi_{\epsilon}(s)=e^{-t p^{2}} h_{\epsilon}(t, \sqrt{t} p)
$$

where $h_{\epsilon}$ is defined in (132) and it is uniformly bounded on $\mathbb{R}_{+} \times \mathbb{R}$. For $Y_{\epsilon}$ we have

$$
\widehat{Y}_{\epsilon}(t, p)=\mathrm{i} p e^{-t p^{2}} \chi_{\epsilon}(t)
$$

Thus

$$
\widehat{J}_{\epsilon}(t, p)=\mathrm{i} \int_{\mathbb{R}} d q(p+q) e^{-t\left((p+q)^{2}+q^{2}\right)} h_{\epsilon}(t, \sqrt{t} q) \chi_{\epsilon}(t)=\frac{\mathrm{i} p}{\sqrt{t}} \widehat{\mathcal{W}}_{\epsilon}(t, \sqrt{t} p)
$$

where

$$
\widehat{\mathcal{W}}_{\epsilon}(t, r)=\int_{\mathbb{R}} d q(1+q / r) e^{-\left((r+q)^{2}+q^{2}\right)} h_{\epsilon}(t, q) \chi_{\epsilon}(t)
$$

$\widehat{\mathcal{W}}_{\epsilon}$ is an entire function in $r$ with

$$
\left|\widehat{\mathcal{W}}_{\epsilon}(t, r)\right| \leq C e^{-c(\operatorname{Re} r)^{2}}
$$

if $|\operatorname{Im} r| \leq 1$ (we used $h(t, q)=h(t,-q)$ ). Hence in particular the inverse Fourier transform $\mathcal{W}_{\epsilon}(t, x)$ is in $L^{1}(\mathbb{R})$ uniformly in $t$. We end up with the claim with

$$
W_{\epsilon}(z)=\frac{1}{t} \mathcal{W}_{\epsilon}(t, x / \sqrt{t})
$$

(b) It suffices to study $A_{\epsilon}=Y_{\epsilon} * \partial_{x} W_{\epsilon}$. We get

$$
\widehat{A}_{\epsilon}(t, p)=-p^{2} \int_{0}^{t} e^{-(t-s) p^{2}} \chi_{\epsilon}(t-s) \frac{1}{\sqrt{s}} \widehat{W}_{\epsilon}(s, \sqrt{s} p) d s=\frac{1}{\sqrt{t}} \hat{a}_{\epsilon}(t, \sqrt{t} p)
$$


with

$$
\hat{a}_{\epsilon}(t, p)=-p^{2} \int_{0}^{1} e^{-(1-\sigma) p^{2}} \chi_{\epsilon}((1-\sigma) t) \frac{1}{\sqrt{\sigma}} \widehat{W}_{\epsilon}(\sigma t, \sqrt{\sigma} p) d \sigma .
$$

$\hat{a}_{\epsilon}$ is entire satisfying (147) and the claim follows.

(c) These claims follow from

$$
\left|\widehat{\mathcal{W}}_{\epsilon}(t, r)-\widehat{\mathcal{W}}_{\epsilon}^{\prime}(t, r)\right| \leq C e^{-c(\operatorname{Re} r)^{2}} \mathbf{1}_{\left[\frac{1}{2} \epsilon^{2}, 2 \epsilon^{2}\right]}(t)
$$

(d) Let $B_{\epsilon}=\partial_{x} \mathfrak{C}_{\epsilon}^{2}=2 \mathfrak{C}_{\epsilon} \partial_{x} \mathfrak{C}_{\epsilon}$. Then

$$
\widehat{B}_{\epsilon}(t, p)=2 \mathrm{i} \int_{\mathbb{R}} d q(p+q) e^{-t\left((p+q)^{2}+q^{2}\right)} h_{\epsilon}(t, \sqrt{t} p) h_{\epsilon}(t, \sqrt{t} q)
$$

Comparing with (146) and noting that

$$
\left|2 h_{\epsilon}(t, \sqrt{t} p)-\chi_{\epsilon}(t)\right| \leq C\left(\mathbf{1}_{\left[\frac{1}{2} \epsilon^{2}, 2 \epsilon^{2}\right]}(t)+\mathbf{1}_{\left[\frac{1}{2}, 2\right]}(t)\right)
$$

we get

$$
\left|J_{\epsilon}(z)-\partial_{x} \mathfrak{C}_{\epsilon}(z)^{2}\right| \leq C\left(\epsilon^{-3} e^{-c|x| / \epsilon} \mathbf{1}_{\left[\frac{1}{2} \epsilon^{2}, 2 \epsilon^{2}\right]}(t)+e^{-c|x|} \mathbf{1}_{\left[\frac{1}{2}, 2\right]}(t)\right) .
$$

In the same way we get

$$
\left|Y_{\epsilon}(z)-2 \partial_{x} \mathfrak{C}_{\epsilon}(z)\right| \leq C\left(\epsilon^{-2} e^{-c|x| / \epsilon} \mathbf{1}_{\left[\frac{1}{2} \epsilon^{2}, 2 \epsilon^{2}\right]}(t)+e^{-c|x|} \mathbf{1}_{\left[\frac{1}{2}, 2\right]}(t)\right) .
$$

\section{REFERENCES}

[1] M. Hairer: A theory of regularity structures. Invent. Math. 198(2), 269-504 (2014)

[2] R. Catellier and K. Chouk: Paracontrolled distributions and the 3-dimensional stochastic quantization equation. ArXiv: 1310.6869 (2013)

[3] P. Gonçalves and M. Jara: Nonlinear fluctuations of weakly asymmetric interacting particle systems. Arch. Rational Mech. Anal. 212(2), 597-644 (2014)

[4] M. Gubinelli, P. Imkeller, and N. Perkowski: Paracontrolled distributions and singular PDEs. Forum Math. Pi. 3 (2015)

[5] A. Kupiainen: Renormalization group and Stochastic PDEs. Ann. Henri Poincaré. 17(3), 497-535 (2016)

[6] H. Spohn: Nonlinear Fluctuating Hydrodynamics for Anharmonic Chains. J. Stat. Phys. 154(5), 1191-1227 (2014)

[7] M. Hairer: Solving the KPZ equation. Ann. Math. 178(2), 559-664 (2013)

[8] K. Wilson: The renormalization group and critical phenomena. Nobel Lecture. Rev. Mod. Phys. (1984)

[9] S.B. Chae: Holomorphy and calculus in normed spaces. Marcel Decker, New York (1985)

[10] J. Bricmont, A. Kupiainen and G. Lin: Renormalization Group and Asymptotics of Solutions of Nonlinear Parabolic Equations. Comm. Pure Appl. Math. 47, 893-922 (1994)

[11] J. Bricmont, K. Gawedzki and A. Kupiainen: KAM theorem and quantum field theory. Comm. Math. Phys. 201(3), 699-727 (1999)

[12] Vladimir I. Bogachev: Gaussian Measures. Mathematical Surveys and Monographs. American Mathematical Society (1998)

[13] D. Nualart: The Malliavin calculus and related topics. Probability and its Applications (New York). Springer-Verlag, Berlin, second ed. (2006)

[14] J. Lukkarinen and M. Marcozzi: Wick polynomials and time-evolution of cumulants. ArXiv: $1503.05851(2016)$

University of Helsinki, Department of Mathematics and Statistics, P.O. Box 68 , FiN00014 University of Helsinki, Finland

E-mail address: antti.kupiainen@helsinki.fi

E-mail address: matteo.marcozzi@helsinki.fi 